\title{
Drosophila Bruton's Tyrosine Kinase Regulates Habituation Latency and Facilitation in Distinct Mushroom Body Neurons
}

\author{
-Ilianna G. Roussou, ${ }^{1,2}$ ๑Katerina Papanikolopoulou, ${ }^{1}$ Charalambos Savakis, ${ }^{1,2}$ and $\odot$ Efthimios M. C. Skoulakis ${ }^{1}$ \\ ${ }^{1}$ Institute for Fundamental Biomedical Research, Biomedical Science Research Centre "Alexander Fleming," Vari 16672, Greece, and 2School of Medicine, \\ University of Crete, Heraklion, 71003, Greece
}

Habituation is the adaptive behavioral outcome of processes engaged in timely devaluation of non-reinforced repetitive stimuli, but the neuronal circuits and molecular mechanisms that underlie them are not well understood. To gain insights into these processes we developed and characterized a habituation assay to repetitive footshocks in mixed sex Drosophila groups and demonstrated that acute neurotransmission from adult $\alpha / \beta$ mushroom body (MB) neurons prevents premature stimulus devaluation. Herein we demonstrate that activity of the non-receptor tyrosine kinase dBtk protein is required within these neurons to prevent premature habituation. Significantly, we also demonstrate that the complementary process of timely habituation to the repetitive stimulation is facilitated by $\alpha^{\prime} / \beta^{\prime} \mathrm{MB}$ neurons and also requires $\mathrm{dBtk}$ activity. Hence our results provide initial insights into molecular mechanisms engaged in footshock habituation within distinct MB neurons. Importantly, dBtk attenuation specifically within $\alpha^{\prime} / \beta^{\prime}$ neurons leads to defective habituation, which is readily reversible by administration of the antipsychotics clozapine and risperidone suggesting that the loss of the kinase may dysregulate monoamine receptors within these neurons, whose activity underlies the failure to habituate.

Key words: antipsychotics; Btk; Drosophila; habituation; mushroom bodies

\section{Significance Statement}

Habituation refers to processes underlying decisions to attend or ignore stimuli, which are pivotal to brain function as they underlie selective attention and learning, but the circuits involved and the molecular mechanisms engaged by the process therein are poorly understood. We demonstrate that habituation to repetitive footshock involves two phases mediated by distinct neurons of the Drosophila mushroom bodies and require the function of the dBtk non-receptor tyrosine kinase. Moreover, habituation failure upon dBtk abrogation in neurons where it is required to facilitate the process is readily reversible by antipsychotics, providing conceptual links to particular symptoms of schizophrenia in humans, also characterized by habituation defects and ameliorated by these pharmaceuticals.

\section{Introduction}

Habituation is a form of adaptive behavioral plasticity that permits animals to ignore repetitive or prolonged non-reinforced

Received March 20, 2019; revised Aug. 19, 2019; accepted Aug. 21, 2019.

Author contributions: E.M.C.S. and I.G.R. designed research; I.G.R. and K.P. performed research; C.S. contributed unpublished reagents/analytic tools; I.G.R. and E.M.C.S. analyzed data; I.G.R., K.P., C.S., and E.M.C.S. wrote the paper.

This work was co-financed by the European Union (European Social Fund) and Greek national funds through the Operational Program "Education and Lifelong Learning" of the National Strategic Reference Framework 2007-2013, Research Funding Program: THALES- Investing in Knowledge Society through the European Social Fund, Project: “THALES-BSRC 'Alexander Fleming'-Development and employment of Minos-based genetic and functional genomic technologies in model organisms (MINOS)"-MIS: 376898; and by the project "Strategic Development of the Biomedical Research Institute 'Alexander Fleming'" (MIS 5002562), which is implemented under the "Action for the Strategic Development on the Research and Technological Sector", funded by the Operational Programme "Competitiveness, Entrepreneurship and Innovation" (NSRF 2014-2020) and co-financed by Greece and the European Union (European Regional Development Fund); and partly by Fondation Sante. We thank the Bloomington stimuli (Thompson and Spencer, 1966; Rankin et al., 2009). Timely devaluation of such stimuli is the behavioral output of largely undefined molecular processes, apparently engaging multiple cellular systems (Ramaswami, 2014). Because habituation underlies gated selective attention and discrimination between novel and pre-experienced stimuli (Gillberg, 2003), it represents a form of adaptive behavioral flexibility, reported defective in patients with migraines (Siniatchkin et al., 2003; Kalita et al.,

Drosophila Stock Center for strains and resources, Daisuke Yamamoto for mutant strains, Flybase for essential information, and Dr. A. Kanellopoulos for performing pilot experiments indicating that Btk mutants failed to habituate.

The authors declare no competing financial interests.

Correspondence should be addressed to Efthimios M. C. Skoulakis at skoulakis@fleming.gr.

https://doi.org/10.1523/JNEUROSCI.0633-19.2019

Copyright $\odot 2019$ the authors 
2014), attention deficit hyperactivity disorder (Jansiewicz et al., 2004; Massa and O'Desky, 2012), schizophrenia (SD; Braff et al., 2001; Meincke et al., 2004) and autism spectrum disorders (ASD; D'Cruz et al., 2013; Tei et al., 2018). Hence, defining molecular mechanisms that govern habituation is likely to contribute toward understanding the molecular etiology of these conditions.

To explore habituation mechanisms of CNS circuits in Drosophila, we developed a novel habituation paradigm to repetitive mild electric footshock (Acevedo et al., 2007). Habituation to repetitive footshock requires structurally and functionally intact mushroom bodies (MBs), neurons also essential for associative learning and memory (Guven-Ozkan and Davis, 2014). The MBs are comprised by $\sim 2000$ neurons per hemisphere subdivided into $\alpha / \beta, \alpha^{\prime} / \beta^{\prime}$ and $\gamma$ subtypes (Crittenden et al., 1998). Because neurotransmission from $\alpha / \beta \mathrm{MB}$ neurons is required to suppress premature habituation, we proposed that physiologically, it blocks devaluation of the repetitive footshock stimuli, hence required for habituation latency (Acevedo et al., 2007). However, whether other intrinsic or extrinsic $\mathrm{MB}$ neurons are required to facilitate habituation remained unclear.

To address this question and to identify proteins governing habituation within the MBs, we have conducted a genetic screen for mutants that present defective habituation. Here we report on the role of one of the proteins identified in the screen, Bruton's tyrosine kinase (dBtk), which belongs to the Src non-receptor kinase superfamily and is associated with agammaglobulinemia in humans (Mattsson et al., 1996). The Drosophila dBtk29A (dBtk) gene encodes two proteins by alternative splicing, dBtk type 1 and dBtk type 2, with the latter considered orthologous to the human protein (Gregory et al., 1987). dBtk consists of conserved $\mathrm{SH} 2, \mathrm{SH} 3$ and kinase domains, whereas an $\mathrm{N}$-terminal plekstrin homology $(\mathrm{PH})$ domain characterizes the larger type 2 protein (Tsikala et al., 2014). dBtk is implicated in many essential functions in Drosophila (Gregory et al., 1987; Roulier et al., 1998; Baba et al., 1999; Hamada-Kawaguchi and Yamamoto, 2017), including regulation of the actin cytoskeleton (Tsikala et al., 2014). Although dBtk is highly expressed in the fly CNS, there is limited information regarding its functional role(s) therein (Asztalos et al., 2007; Sunouchi et al., 2016). Here we demonstrate acute differential roles for this kinase within distinct MB neuronal populations in the regulation of habituation dynamics to repeated footshock stimuli.

\section{Materials and Methods}

\section{Drosophila culture and strains}

Drosophila were cultured in standard wheat-flour-sugar food supplemented with soy flour and $\mathrm{CaCl}_{2}$ (Acevedo et al., 2007) at $18^{\circ} \mathrm{C}$ or $25^{\circ} \mathrm{C}$. All MiMIC insertions were from the Bloomington Stock Center (BDRC; Indiana University; Venken et al., 2011) and they were backcrossed to $y^{l} w^{1}$ for at least seven generations before use in behavioral experiments. MBGal80 (Krashes et al., 2007) was obtained from Ron Davis (Scripps Florida). The Btk-Gal4 (49182), dncGal4 (48571), and Btk RNAi stocks (35159 and 25791) were from BDRC. To generate the driver heterozygote controls for experiments with the RNAi-encoding transgenes, driverbearing strains were crossed to their $y^{I} v^{1}$ (BDSC, 36303) background. The UAS-Btk lines (109-093 and 109-095) were from the Kyoto Stock Center (Kyoto Institute of Technology). VT44966-Gal4 ( $\gamma$-driver) was from the Vienna Drosophila Resource Center (VDRC; Vienna Biocenter Core Facilities, 203571). The $\alpha^{\prime} / \beta^{\prime}$ Gal4 drivers VT030604 (VDRC, 200228 ) and c305a were a kind gift from S. Waddell (University of Oxford). The glial driver repo-Gal4, the pan-neuronal drivers elav-Gal4 and Ras2Gal4, and the mushroom body specific drivers 247-Gal4, leo-Gal4, c739-Gal4, c772-Gal4 were described previously (Aso et al., 2009; Gouzi et al., 2018). The Gal80 ${ }^{\text {ts }}$ transgene was added to the driver-bearing chromosomes by recombination or standard crosses as indicated.
Description of Gal4 expression patterns used in this work.

Elav: pan-neuronal expression in all developmental stages (FlyBase ID: FBrf0237128)

Ras2: throughout the larval and adult CNS, enriched in the adult MBs (Gouzi et al., 2011)

repo: all glia (FlyBase ID: FBrf0237128)

leo: adult $\alpha, \alpha^{\prime}, \beta, \beta^{\prime}, \gamma \mathrm{MB}$ neurons (Messaritou et al., 2009)

247: adult $\alpha, \alpha^{\prime}, \beta, \beta^{\prime}, \gamma \mathrm{MB}$ neurons (RRID:BDSC_50742)

$d n c$ : adult $\alpha, \alpha^{\prime}, \beta, \beta^{\prime}, \gamma \mathrm{MB}$ neurons, scattered neurons in subesophageal ganglion and ventral optic lobes (Gai et al., 2016)

c772: adult $\alpha, \beta, \gamma \mathrm{MB}$ neurons, antennal lobe, medulla, tritocerebrum (Aso et al., 2009)

c739: adult $\alpha, \beta \mathrm{MB}$ neurons, antennal lobe, medulla, restricted protocerebral neurons, inferior neuropils (Aso et al., 2009).

VT44966: $\gamma$ MB neurons, wedge neurons, superior lateral protocerebrum, gnathal neurons, medial bundle (Shyu et al., 2017)

c305a: adult $\alpha^{\prime}, \beta^{\prime} \mathrm{MB}$ neurons, antennal nerve, medulla, restricted protocerebral neurons, inferior neuropil, gnathal neurons (Aso et al., 2009)

VT030604: adult $\alpha^{\prime}, \beta^{\prime}$ MB neurons, gnathal neurons (Shyu et al., 2017)

\section{Behavioral assays}

All flies used in behavioral experiments were tested 3-5 d after emergence. All experiments were performed under dim red light at $25^{\circ} \mathrm{C}$ and $65-75 \%$ relative humidity. To obtain animals for behavioral experiments Gal4 driver homozygotes were crossed en masse to strains carrying either UAS-btk, UAS-btk-RNAi, or UAS-shi ${ }^{\text {ts }}$ transgenes. Animals expressing Gal80 ${ }^{\text {ts }}$ (McGuire et al., 2003) were raised at $18^{\circ} \mathrm{C}$ until hatching and then placed at $30^{\circ} \mathrm{C}$ for $2 \mathrm{~d}$ before testing. Flies carrying UAS-shi ${ }^{\text {ts }}$ were reared at $18^{\circ} \mathrm{C}$ and the dynamin was inactivated by incubation at $32^{\circ} \mathrm{C}$ for 30 min before the behavioral experiment.

Electric footshock avoidance. Experiments were performed as described before (Acevedo et al., 2007). Briefly, $\sim 70$ flies were placed at the choice point of a T-maze to choose for $90 \mathrm{~s}$ between an electrified and an otherwise identical inert standard copper grid. In the electrified grid, $45 \mathrm{~V}$ shocks were delivered every $5.15 \mathrm{~s}$, each lasting $1.2 \mathrm{~s}$. The avoiding fraction (AF) was calculated by dividing the number of flies avoiding the shock by the total number of flies.

Habituation to electric footshock. Habituation to electric shock experiments were performed as described before (Acevedo et al., 2007). Briefly, for the training phase $\sim 70$ flies were sequestered in the upper arm of a standard T-maze lined with an electrifiable grid. They were exposed to 15 $1.2 \mathrm{~s}$ electric shocks at $45 \mathrm{~V}$ with a $5.15 \mathrm{~s}$ interstimulus interval. Air was not drawn through the tube during training to avoid association of the shocks with air. After a $30 \mathrm{~s}$ rest and $30 \mathrm{~s}$ for transfer to the lower part of the maze, the flies were tested by choosing between an electrified and an inert grid. Therefore, the earliest measures of post-training responses are $1 \mathrm{~min}$ after the flies received the last training stimulus. Testing was performed at the same voltage $(45 \mathrm{~V})$ as for training. During the $90 \mathrm{~s}$ choice period, 17-18 $1.2 \mathrm{~s}$ stimuli were delivered to the electrified arm of the maze. At the end of the choice period, the flies in each arm were trapped and counted, and the habituation fraction (HF) was calculated by dividing the number of flies preferring the shock by the total number of flies, as above. Finally, the habituation index (HI) was calculated as (HF $\mathrm{AF}) \times 100 \%$ and therefore represents the change in footshock avoidance contingent upon prior footshock experience (habituation). Although the absolute avoidance score is variable, even for the same genotypes (Table 1 ), as expected for behavioral experiments performed over a significant time period, because the $\mathrm{HI}$ measures the relative change in avoidance within each genotype, it is not affected by such variability. In fact, failure to habituate, which is the primary phenotype reported herein, is a manifestation of maintained avoidance relative to that of naive flies of the same genotype.

Dishabituation. To distinguish habituation from fatigue or sensory adaptation, flies were dishabituated post-training with an $8 \mathrm{~s}$ puff [yeast odor (YO)] of air drawn at $500 \mathrm{ml} / \mathrm{min}$ over a $30 \%(\mathrm{w} / \mathrm{v}$ ) aqueous solution of Brewer's yeast (Acros Organics) and then were submitted to testing. 
Table 1. Collective avoidance indices and statistics

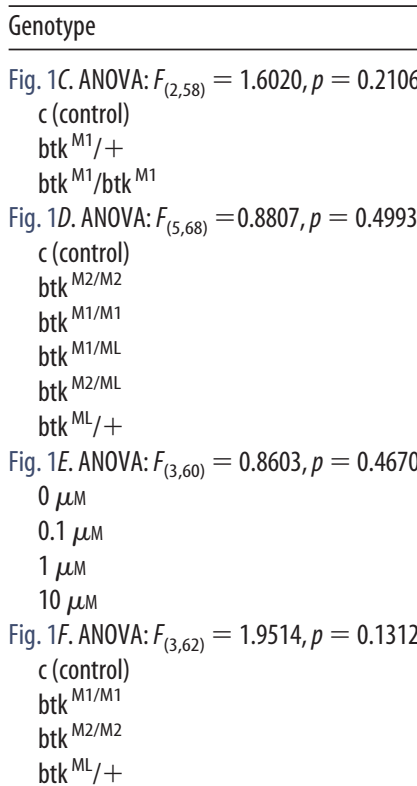

Fig. 2A. $\operatorname{ANOVA:} \mathrm{F}_{(2,44)}=1.3353, p=0.2740$ elavGAL4/+;UAS-btk ${ }^{R N A T} /$ GAL $^{\text {ts }}$

elavGAL $4 /+; G A L 80$ ts $/+$ UASbtk $k^{\text {NNAiT }} /+$

Fig. 2B. ANOVA: $\mathrm{F}_{(2,35)}=2.0785, p=0.1412$ elavGAL4/+;UAS-btk ${ }^{\text {RNAi2 }} /{\text { GAL } 80^{\text {ts }}}^{\text {ts }}$ elavGAL $4 /+; G A L 80^{\text {ts }} /+$ UASbtkNAi2/+

Fig. 2C. ANOVA: $_{(2,30)}=1.100 p=0.3468$ elavGAL4/+;UAS-btk ${ }^{\text {RNAit }} /$ GAL $0^{\text {ts }}$ elavGAL $4 /+; G A L 80^{\text {ts }} /+$ UASbtK $^{\text {RNAi-1 }} /+$

Fig. 2D. ANOVA: $F_{(2,27)}=0.1719, p=0.8431$ Ras2GAL4,GAL80 ${ }^{\text {t5 }} /+$; UASbtk ${ }^{\text {RNAir }}$ Ras2GAL4, GAL80 ${ }^{\text {ts }} /+$ UASBTK $K^{\text {RAAi }} /+$

Fig. 2E. ANOVA: $\mathrm{F}_{(2.46)}=2.1609, p=0.1273$ repoGAL4,GAL $80^{\text {ts }} /$ UAS-btk RAAiT $^{\text {RN }}$ repoGAL4,GAL80 ${ }^{\text {ts }} /+$ $\mathrm{UASbtk}^{\text {RNAiT }} /+$

Fig. 2F. ANOVA: $\mathrm{F}_{(2,47)}=3.2093, p=0.0498$ elavGAL4/+;MBG80,GAL80 ${ }^{\mathrm{t}} / \mathrm{UASb}^{\text {RNAi1 }}$ elavGAL4/+;MBG80, GAL80 ${ }^{\text {ts }} /+$ UASbtk ${ }^{\text {RNAir }} /+$

Fig. 3A. ANOVA: $F_{(2,34)}=0.5313, p=0.5929$ leoMBGAL4,GAL80 ${ }^{\text {ts }}$;UAS-btk ${ }^{\text {RNAi1 }} /+$ leoMB-GAL4, GAL 80 ts $/+$ UAS-btk ${ }^{R N A i 1} /+$

Fig. 3B. ANOVA: $F_{(5,66)}=2.7712, p=0.0255$ leoMBGAL4, btk ${ }^{\mathrm{M1}} /+;$ GAL80ts/ + UN leoMBGAL4,btk ${ }^{\mathrm{M} 1} /$ btk $^{\mathrm{M} 1} ;$ GAL80ts/ + UN

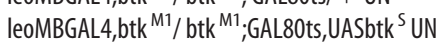
leoMBGAL4,btk ${ }^{\mathrm{M} 1} /+$; GAL80ts/ + IN leoMBGAL4,btk ${ }^{\mathrm{M} 1} /$ btk $^{\mathrm{M} 1} ;$ GAL80ts/ + IN

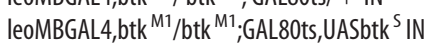

Fig. 3C. ANOVA: $F_{(2,39)}=0.5892, p=0.5599$ leoMBGAL4/+;UASbtk ${ }^{\text {NNAiz }} /{\text { GAL } 80^{\text {ts }}}^{\text {ts }}$ leoMBGAL $4 /+;$ GAL 80 ts $/+$ UAS-btk ${ }^{R N A i 2} /+$

Fig. 3D. ANOVA: $F_{(2,42)}=0.4747, p=0.6256$ 247MBGAL4,GAL80 ${ }^{\mathrm{t}} /$ UAS- $b t k^{\text {RNAiT }}$ 247MBGAL4,GAL $80^{\text {t5 }} /+$ UASbtk ${ }^{\text {RNAir }} /+$

Mean \pm SEM $t$ Ratio $p$

$0.76 \pm 0.03$

$0.77 \pm 0.05$

$0.66+0.07$

$0.61 \pm 0.02$

$0.62 \pm 0.04$

$0.59 \pm 0.05$

$0.55 \pm 0.05$

$0.53 \pm 0.05$

$0.63 \pm 0.03$

$0.53 \pm 0.03$

$0.49 \pm 0.03$

$0.55 \pm 0.03$

$0.49 \pm 0.04$

$0.54 \pm 0.04$

$0.67 \pm 0.05$

$0.51 \pm 0.06$

$0.62 \pm 0.06$

$0.59 \pm 0.05$

$0.61 \pm 0.04$

$0.49 \pm 0.07$

$-0.237$

1.3101

0.8139

$0.66 \pm 0.04$

$0.72 \pm 0.03$

$0.78 \pm 0.03$

$-1.105$

$-2.006$

0.2771

$0.73 \pm 0.05$

$0.63 \pm 0.03$

$0.68 \pm 0.05$

$1.4796 \quad 1.1502$

$0.7939 \quad 0.4340$

$0.67 \pm 0.08$

$0.65 \pm 0.05$

$0.70 \pm 0.04$

$0.1811 \quad 0.8578$

$-0.386 \quad 0.703$

$0.63 \pm 0.05$

$0.73 \pm 0.03$

$0.67 \pm 0.03$

$\begin{array}{ll}-2.075 & 0.044\end{array}$

$-0.955 \quad 0.3449$

$0.71 \pm 0.02$

$0.63 \pm 0.02$

$0.71 \pm 0.03$

$0.46 \pm 0.09$

$0.56 \pm 0.07$

$0.56 \pm 0.07$

$0.65 \pm 0.03$

$0.50 \pm 0.03$

$0.59 \pm 0.05$

$0.56 \pm 0.04$

$0.45 \pm 0.04$

$0.62 \pm 0.04$

$0.65 \pm 0.05$

$0.68 \pm 0.03$

$0.61 \pm 0.06$

$0.63 \pm 0.03$

$0.60 \pm 0.02$

$0.61 \pm 0.03$

$\begin{array}{ll}2.2266 & 0.031\end{array}$

$\begin{array}{ll}-0.085 & 0.932\end{array}$

$\begin{array}{rr}-0.905 & 0.3723\end{array}$

$\begin{array}{ll}-0.892 & 0.3788\end{array}$

$2.205 \quad 0.031$

$0.9337 \quad 0.354$

$\begin{array}{ll}-1.265 & 0.211\end{array}$

$-0.914 \quad 0.364$

$\begin{array}{ll}-0.471 \quad 0.640 \\ 0.6403 & 0.5260\end{array}$

$0.6403 \quad 0.5260$

$\begin{array}{ll}0.9688 & 0.3385\end{array}$

$0.5817 \quad 0.5640$
Table 1. Continued

Fig. 3E. ANOVA: $F_{(2,30)}=0.2194, p=0.8044$

dncGAL4,GAL80 ts $/$ UASbtk $^{\text {RNAit }}$

dncGAL4,GAL $80^{\text {ts }} /+$

UASbtk ${ }^{\text {RNAir }} /+$

Fig. 3F. ANOVA: $F_{(2,44)}=1.1638, p=0.3222$ C772GAL4,GAL $80^{\text {ts }} /$ UASbtk $^{\text {RAAiT }}$

C772GAL4,GAL80 ${ }^{\text {ts }} /+$

UASbtk ${ }^{\text {RNAiT }} /+$

Fig. 3G. ANOVA: $F_{(2,43)}=1.891, p=0.1766$ UASbtk ${ }^{\text {RNAi- } 1} / \mathrm{C}^{2} 339 \mathrm{GAL} 4, \mathrm{GAL} 80^{\text {ts }}$

C739GAL4,GAL80 5 / +

UASbtk ${ }^{\text {RAAiT }} /+$

Fig. 3H. ANOVA: $F_{(2,47)}=3.0762, p=0.0559$ $\gamma$ lobeGAL4/+;UASbtk ${ }^{\text {RNAiT }} /{\text { GAL } 800^{\text {ts }}}$

$\gamma$ lobe GAL $4 /+; G A L 80$ ts $/+$

UASbtk ${ }^{\text {RNAiT }} /+$

Fig. 3I. ANOVA: $F_{(2,28)}=1.0786, p=0.3548$ c305aGAL $4 /+;$ UASbtk RNAii $^{\prime} /$ GAL $0^{\text {ts }}$

C305a GAL4/+;GAL80 5 / + UASbtk ${ }^{\text {RNAiT }} /+$

Fig. 3J. ANOVA: $F_{(2,49)}=2.5477, p=0.0156$ VT030604GAL $4 /+$, UASbtk ${ }^{\text {RNAi1 }} 18^{\circ} \mathrm{C}$ VT030604GAL4/+, UASbtk ${ }^{\text {RNAi1 }} 25^{\circ} \mathrm{C}$ VT030604-GAL4/+, UAS-btk ${ }^{\text {RNAir }} 18>30^{\circ} \mathrm{C}$

Fig. $3 K$. ANOVA: $F_{(1,25)}=9.7589, p=0.0046$ C305aGAL4-UAS shits UN C305aGAL4-UAS shis IN

Fig. 4A. ANOVA: $F_{(5,104)}=4.7413, p=0.0006$ control 6 sh

btk $^{\mathrm{M} 1 / \mathrm{M} 1} 6$ sh

control 10 sh

btk $^{\mathrm{M} 1 / \mathrm{M} 1} 10 \mathrm{sh}$

control 15 sh

btk $^{\mathrm{M} 1 / \mathrm{M} 1} 15 \mathrm{sh}$

Fig. 4B. ANOVA: $F_{(8,156)}=2.8880, p=0.0051$ UASbtk ${ }^{\text {RNAiT }} /$ elavGAL4; GAL80 ${ }^{\text {ts }} 6$ sh

elavGAL4;GAL80 ${ }^{\text {ts }} /+6$ sh

UAS-btk ${ }^{R N A i-1} /+6 s h$

UASbtk ${ }^{\text {RNAir }} /$ elavGAL4;GAL80 $80^{\text {ts }} 10$ sh

elavGAL4;GAL $80^{\text {ts }} \%+10$ sh

UAS btk ${ }^{\text {RNAiT }} /+10$ sh

UASbtk $^{\text {RNAir } / \text { elavGAL4; GAL80 }}$ ts 15 sh

elavGAL4;GAL80 ${ }^{\text {ts }} /+15$ sh

UASbtk $k^{\text {RNAi-1 }} /+15$ sh

Fig. 4C. ANOVA: $F_{(2,28)}=1.2620, p=0.2999$ $0 \mu \mathrm{m}$

$0.1 \mu \mathrm{M}$

$1 \mu \mathrm{M}$

Fig. 4D. ANOVA: $F_{(2,30)}=0.0147, p=0.9854$ Habituation, 15 shocks

Y0 dishabituation

AVD after yeast puff (Table continues.)

Fig. 4E. ANOVA: $F_{(2,30)}=0.0147, p=0.9854$ btk $^{\mathrm{M} 1 / \mathrm{M} 1}$ habituation $6 \mathrm{sh}$

btk ${ }^{\mathrm{M} 1 / \mathrm{M} 1}$ Dishabituation 6 sh

btk $^{\mathrm{M1} / \mathrm{M} 1}$ habituation $10 \mathrm{sh}$

btk ${ }^{\mathrm{M} 1 / \mathrm{M} 1}$ Dishabituation 10 sh

btk ${ }^{\mathrm{M} 1 / \mathrm{M} 1} \mathrm{AVD}$ after yeast puff

Fig. 5A. ANOVA: $F_{(5,119)}=3.8885 p=0.0027$ leoGAL4/+;UASbtk ${ }^{\text {RAiT }} /$ GAL $80^{\text {ts }}$ UN 6 sh

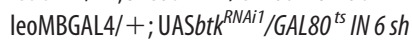
leoMBGAL4/+; UASbtk ${ }^{\text {RNAir }} /$ GAL $^{\text {ts }}$ IN 10 sh

leoMBGAL4/+; UASbtk RNAiT $/$ GAL8O $^{\text {ts }}$ IN 15 sh
Mean \pm SEM $t$ Ratio $\quad p$

$0.53 \pm 0.02$

$0.54 \pm 0.03 \quad-0.198 \quad 0.8443$

$0.63 \pm 0.03$

$\begin{array}{lll}0.54 \pm 0.06 & 1.451 & 0.1542\end{array}$

$0.57 \pm 0.03 \quad 1.1173 \quad 0.2702$

$0.57 \pm 0.05$

$0.46 \pm 0.04$

$0.53 \pm 0.04$

$\begin{array}{ll}1.8657 & 0.0692\end{array}$

$0.6998 \quad 0.488$

$0.58 \pm 0.03$

$0.49 \pm 0.03$

$0.59 \pm 0.03$

$\begin{array}{rr}2.0924 & 0.0421\end{array}$

$0.77 \pm 0.03$

$0.71 \pm 0.02$

$0.77 \pm 0.04$

$\begin{array}{ll}1.3002 & 0.2049\end{array}$

$0.0361 \quad 0.9715$

$0.74 \pm 0.03$

$0.67 \pm 0.02$

$0.78 \pm 0.03$

$\begin{array}{ll}1.9623 & 0.0557\end{array}$

$\begin{array}{ll}-0.972 & 0.3361\end{array}$

$0.85 \pm 0.03$

$0.75 \pm 0.01$

$-3.124$

0.0046

$0.79 \pm 0.04$

$0.63 \pm 0.04$

$0.69 \pm 0.03$

$0.60 \pm 0.04$

$2.9535 \quad \mathbf{0 . 0 0 3 9}$

$0.73 \pm 0.02$

$0.58 \pm 0.04$

1.6587

0.1003

$2.9818 \quad 0.0036$

$0.82 \pm 0.02$

$0.66 \pm 0.03$

$0.71 \pm 0.04$

$0.77 \pm 0.02$

$0.66 \pm 0.03$

$0.66 \pm 0.06$

$0.73 \pm 0.04$

$0.74 \pm 0.03$

$0.70 \pm 0.03$

$0.6554 \quad 0.5132$

$0.58 \pm 0.05$

$0.64 \pm 0.04$

$0.68 \pm 0.04$

$-0.832$

0.4129

$0.76 \pm 0.02$

$0.77 \pm 0.02$

$0.77 \pm 0.02$

$-0.147$

0.8839

$0.68 \pm 0.03$

$0.68 \pm 0.03$

$0.73+0.04$

$0.73 \pm 0.03$

$0.73 \pm 0.04$

\begin{tabular}{ll}
0.0617 & 0.9511 \\
\hline
\end{tabular}

$0.81 \pm 0.02$

$0.78 \pm 0.02$

$0.82 \pm 0.01$

leoMBGAL4/+; UASbtk ${ }^{\text {RNAii } / G A L 80^{\text {ts }} \text { UN } 10 \text { sh }}$

leoMB-GAL4/+; UASbtk ${ }^{\text {RNAir }} /$ GAL $80^{\text {ts }}$ UN 15 sh

$0.75 \pm 0.02$

$0.82 \pm 0.01$

$0.75 \pm 0.02$ 


\section{Table 1. Continued}

\begin{tabular}{|c|c|c|c|}
\hline Genotype & Mean \pm SEM & $t$ Ratio & $p$ \\
\hline \multicolumn{4}{|l|}{ Fig. 5B. ANOVA: $F_{(5,77)}=0.2798, p=0.9227$} \\
\hline 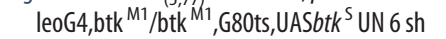 & $0.69 \pm 0.03$ & & \\
\hline leoG4,btk ${ }^{\mathrm{M} 1} / \mathrm{btk}^{\mathrm{M} 1}, \mathrm{G} 80 \mathrm{ts}$, UASbtk IN 6 sh & $0.71 \pm 0.03$ & -0.391 & 0.6966 \\
\hline \multicolumn{4}{|c|}{ leoG4,btk ${ }^{\mathrm{M1}} / \mathrm{btk}^{\mathrm{M1}}, \mathrm{G} 80 \mathrm{ts}, \mathrm{UAS}-\mathrm{btk}{ }^{\mathrm{S}}$ UN 10 sh $0.69 \pm 0.03$} \\
\hline leoG4,btk ${ }^{\mathrm{M1}} / \mathrm{btk}^{\mathrm{M1}}{ }^{\mathrm{1}}$,Gots,UAS-btk ${ }^{\mathrm{S}}$ IN 10 sh & $0.72 \pm 0.03$ & -0.759 & 0.4505 \\
\hline \multicolumn{4}{|c|}{ leoG4,btk ${ }^{\mathrm{M1}} /$ /btk $^{\mathrm{M1}}, \mathrm{G} 80 \mathrm{ts}$, UAS-btk ${ }^{\mathrm{S}}$ UN 15 sh $0.69 \pm 0.03$} \\
\hline leoG4,btk ${ }^{\mathrm{M} 1} / \mathrm{btk}^{\mathrm{M} 1}, \mathrm{G} 80 \mathrm{ts}$, UAS-btk ${ }^{\mathrm{S}}$ IN 15 sh & $0.72 \pm 0.03$ & -0.759 & 0.4505 \\
\hline \multicolumn{4}{|l|}{ Fig. 5C. ANOVA: $F_{(5,77)}=1.1341, p=0.3503$} \\
\hline UAS-btk ${ }^{\text {RNAir }} /$ c739-GAL4,GAL80 $^{\text {t5 }}$ UN 6 sh & $0.73 \pm 0.02$ & & \\
\hline UAS-btk ${ }^{\text {RNAi1 }} /$ c739-GAL4,GAL80 $^{\text {ts }}$ IN 6 sh & $0.72 \pm 0.02$ & 0.4393 & 0.6617 \\
\hline UAS-btk ${ }^{\text {RNAi1 }} /$ C739-GAL4,GAL80 ${ }^{\text {ts }}$ UN 10 sh & $0.74 \pm 1.01$ & & \\
\hline UAS-btk ${ }^{\text {RAAiT }} / \mathrm{C} 739-G A L 4, G A L 80^{\text {ts }}$ IN 10 sh & $0.70 \pm 0.02$ & 1.2977 & 0.1985 \\
\hline UAS-btk ${ }^{\text {RNAiT }} / \mathrm{c} 739-G A L 4$, GAL80 ${ }^{\text {ts }}$ UN 15 sh & $0.74 \pm 1.01$ & & \\
\hline UAS-btk ${ }^{\text {RNAiT }} /$ c739-GAL4,GAL80 ${ }^{\text {ts }}$ IN 15 sh & $0.70 \pm 0.02$ & 1.2977 & 0.1985 \\
\hline \multicolumn{4}{|l|}{ Fig. 5D. ANOVA: $F_{(5,82)}=1.3274 p=0.2614$} \\
\hline c305aGAL4,GAL80 15 /UAS-btk ${ }^{\text {RNAi1 }}$ UN 6 sh & $0.78 \pm 0.02$ & & \\
\hline c305aGAL4,GAL80 ${ }^{\text {ts }} /$ UAS-btk ${ }^{\text {RNAi1 }}$ IN 6 sh & $0.74 \pm 0.02$ & 1.3687 & 0.1751 \\
\hline c305aGAL4,GAL80 ${ }^{\text {t5 }} /$ UAS-btk ${ }^{\text {RNAi1 }}$ UN 10 sh & $0.78 \pm 0.02$ & & \\
\hline c305aGAL4,GAL80 ${ }^{\text {ts }} /$ UAS-btk ${ }^{\text {RNAi1 }}$ IN 10 sh & $0.74 \pm 0.02$ & 1.5464 & 0.1261 \\
\hline c305aGAL4,GAL80 $0^{\text {ts }} /$ UAS-btk ${ }^{\text {RNAir }}$ UN 15 sh & $0.78 \pm 0.02$ & & \\
\hline c305aGAL4,GAL80 ${ }^{\text {ts }} /$ UAS-btk ${ }^{\text {RAi1 }}$ IN 15 sh & $0.74 \pm 0.02$ & 1.5222 & 1.1321 \\
\hline \multicolumn{4}{|l|}{ Fig. 6 A. ANOVA: $F_{(2,28)}=1.1989, p=0.3176$} \\
\hline $0 \mu \mathrm{M}$ & $0.37 \pm 0.04$ & & \\
\hline $5 \mu \mathrm{M}$ & $0.45 \pm 0.04$ & -1.45 & 0.1591 \\
\hline $10 \mu \mathrm{M}$ & $0.43 \pm 0.03$ & -1.139 & 0.2652 \\
\hline \multicolumn{4}{|l|}{ Fig. $6 B$. ANOVA: $F_{(2,30)}=0.8493, p=0.4384$} \\
\hline $0 \mu \mathrm{M}$ & $0.50 \pm 0.05$ & & \\
\hline $5 \mu \mathrm{M}$ & $0.54 \pm 0.05$ & -0.624 & 0.5376 \\
\hline $10 \mu \mathrm{m}$ & $0.46 \pm 0.03$ & 0.5694 & 0.5736 \\
\hline \multicolumn{4}{|l|}{ Fig. 6 C. ANOVA: $F_{(3,44)}=1.3657, p=0.2667$} \\
\hline $0 \mu \mathrm{M}$ & $0.50 \pm 0.03$ & & \\
\hline $0.1 \mu \mathrm{M}$ & $0.57 \pm 0.06$ & -1.432 & 0.1598 \\
\hline $1 \mu \mathrm{M}$ & $0.56 \pm 0.02$ & -1.373 & 0.1772 \\
\hline $10 \mu \mathrm{M}$ & $0.50 \pm 0.03$ & -0.063 & 0.9502 \\
\hline \multicolumn{4}{|l|}{ Fig. 6D. ANOVA: $F_{(2,26)}=8.8963, p=0.0013^{*}$} \\
\hline $0 \mu \mathrm{M}$ & $0.71 \pm 0.03$ & & \\
\hline $5 \mu \mathrm{M}$ & $0.53 \pm 0.07$ & 2.7508 & 0.0111 \\
\hline $10 \mu \mathrm{M}$ & $0.78 \pm 0.03$ & -1.216 & 0.0243 \\
\hline \multicolumn{4}{|l|}{ Fig. $6 E \cdot$ ANOVA: $F_{(2,27)}=1.3673, p=0.2732$} \\
\hline c305aGAL4/+;UAS-btk ${ }^{\text {RNAiT }} /{\text { GAL } 80^{\text {ts }}}$ & $0.66 \pm 0.04$ & & \\
\hline 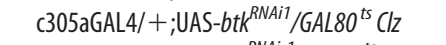 & $0.73 \pm 0.03$ & -1.54 & 0.1361 \\
\hline c305-GAL4/+;UAS-btk ${ }^{\text {RNAi-1 }} /{\text { GAL } 80^{\text {ts }} \text { Ris }}$ & $0.72 \pm 0.03$ & -1.26 & 0.2193 \\
\hline \multicolumn{4}{|l|}{ Fig. 6F. ANOVA: $\mathrm{F}_{(2,26)}=2.2671, p=0.1254$} \\
\hline C739GAL4/+;UAS-btk ${ }^{\text {RNAii }} /$ GAL80 $^{\text {ts }}$ & $0.66 \pm 0.04$ & & \\
\hline 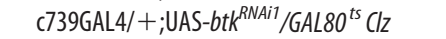 & $0.73 \pm 0.03$ & 1.6255 & 0.1171 \\
\hline 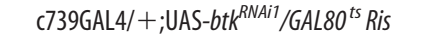 & $0.72 \pm 0.03$ & -0.378 & 0.7085 \\
\hline \multicolumn{4}{|l|}{ Fig. 6G. ANOVA: $F_{(5,95)}=1.8751, p=0.1065$} \\
\hline btk ${ }^{\mathrm{M1} / \mathrm{M} 1} 6 \mathrm{sh}^{\circ}$ & $0.44 \pm 0.05$ & & \\
\hline btk ${ }^{\mathrm{M} 1 / \mathrm{M} 1} \mathrm{Clz} 6 \mathrm{sh}$ & $0.43 \pm 0.02$ & 0.3073 & 0.7593 \\
\hline btk ${ }^{\mathrm{M} 1 / \mathrm{M} 1} 10$ sh & $0.43 \pm 0.05$ & & \\
\hline btk ${ }^{\mathrm{M} 1 / \mathrm{M} 1} \mathrm{Clz} 10$ sh & $0.45 \pm 0.03$ & -0.352 & 0.726 \\
\hline btk ${ }^{\mathrm{M} 1 / \mathrm{M} 1} 15 \mathrm{sh}$ & $0.20 \pm 0.04$ & & \\
\hline btk ${ }^{\mathrm{M} 1 / \mathrm{M} 1} \mathrm{Clz} 15$ sh & $0.43 \pm 0.03$ & -2.527 & 0.0133 \\
\hline \multicolumn{4}{|c|}{ Fig. $6 \mathrm{H}$. ANOVA: $F_{(5,73)}=28.6620, p<0.0001^{*}$} \\
\hline btk ${ }^{\mathrm{M1} / \mathrm{M} 1} 6 \mathrm{sh}$ & $0.35 \pm 0.03$ & & \\
\hline btk ${ }^{\mathrm{M} 1 / \mathrm{M} 1}$ Ris 6 sh & $0.63 \pm 0.03$ & -6.509 & $1.1 \times 10^{-8}$ \\
\hline btk ${ }^{\mathrm{M} 1 / \mathrm{M} 1} 10 \mathrm{sh}$ & $0.36 \pm 0.02$ & & \\
\hline btk ${ }^{\mathrm{M} 1 / \mathrm{M} 1}$ Ris 10 sh & $0.64 \pm 0.04$ & -6.91 & $2.1 \times 10^{-9}$ \\
\hline btk ${ }^{\mathrm{M} 1 / \mathrm{M} 1} 15 \mathrm{sh}$ & $0.38 \pm 0.03$ & & \\
\hline btk ${ }^{\mathrm{M} 1 / \mathrm{M} 1}$ Ris 15 sh & $0.68 \pm 0.04$ & -6.64 & $6.3 \times 10^{-9}$ \\
\hline \multicolumn{4}{|l|}{ Fig. 6l. ANOVA: $F_{(3,52)}=0.5727, p=0.6356$} \\
\hline $0 \mu \mathrm{m} 6 \mathrm{sh}$ & $0.76 \pm 0.03$ & & \\
\hline $10 \mu \mathrm{m} 6 \mathrm{sh}$ & $0.72 \pm 0.03$ & 0.952 & 0.3458 \\
\hline $0 \mu \mathrm{m} 15 \mathrm{sh}$ & $0.78 \pm 0.0 .02$ & & \\
\hline $10 \mu \mathrm{m} 15 \mathrm{sh}$ & $0.73 \pm 0.04$ & 0.8233 & $\begin{array}{l}0.4143 \\
\text { (Table co }\end{array}$ \\
\hline
\end{tabular}

Table 1. Continued

\begin{tabular}{|c|c|c|c|}
\hline Genotype & Mean \pm SEM & $t$ Ratio & $p$ \\
\hline \multicolumn{4}{|c|}{ Fig. 6l. ANOVA: $F_{(3,53)}=65.5164, p<0.0001^{*}$} \\
\hline $0 \mu \mathrm{m} 6 \mathrm{sh}$ & $0.78 \pm 0.01$ & & \\
\hline $10 \mu \mathrm{m} 6 \mathrm{sh}$ & $0.85 \pm 0.00$ & -10.26 & $7 \times 10^{-14}$ \\
\hline $0 \mu \mathrm{M} 15 \mathrm{sh}$ & $0.79 \pm 0.01$ & & \\
\hline $10 \mu \mathrm{m} 15 \mathrm{sh}$ & $0.84 \pm 0.00$ & -9.555 & $7 \times 10^{-13}$ \\
\hline
\end{tabular}

Avoidance of $45 \mathrm{~V}$ footshocks are shown for all genotypes per figure as indicated. Initial ANOVA: values are shown as well as individual statistical comparisons with the respective controls. Significant differences uncovered are highlighted in bold.

Olfactory habituation. Olfactory habituation experiments were performed as detailed in Semelidou et al. (2018). Avoidance of the aversive odorant 3 -octanol (OCT) carried in an airstream of $500 \mathrm{ml} / \mathrm{min}$ in one arm of a standard T-maze versus air was assessed and an index $(\mathrm{AF})$ was calculated. For the "training phase", $\sim 70$ flies were exposed in the upper arm of a standard T-maze to OCT for $4 \mathrm{~min}$. After a $30 \mathrm{~s}$ rest period, the flies were lowered to the center of the maze for testing their osmotactic response by a choice of air versus OCT. At the end of the $90 \mathrm{~s}$ choice period the flies in each arm were trapped counted and HF and HI were calculated as described.

\section{Pharmaceutical treatments}

The Btk inhibitor Ibrutinib (Selleck Chemicals) and the antipsychotic drugs clozapine (Sigma-Aldrich) and risperidone (Tocris Bioscience) were diluted in DMSO and mixed at the indicated final concentrations in Brewer's yeast (Acros Organics) aqueous paste. The concentration ranges used bracketed analogous concentrations as used for humans. Ibrutinib was used at $0.1,1$, and $10 \mu \mathrm{M}$; clozapine at 5 and $10 \mu \mathrm{M}$; and risperidone at $0.1,1$, and $10 \mu \mathrm{M}$. Flies were starved for $5 \mathrm{~h}$ in empty vials at $25^{\circ} \mathrm{C}$ before exposure to drug or vehicle-only containing yeast paste for $14-16 \mathrm{~h}$. The following day, flies were transferred in normal food vials, trained, and tested as detailed for footshock habituation.

\section{Western blots}

Five adult female heads $1-3 \mathrm{~d}$ post-eclosion were homogenized in $1 \times$ Laemmli buffer (50 mm Tris, $\mathrm{pH}$ 6.8, $100 \mathrm{~mm}$ DTT, 5\% 2-mercaptoethanol, $2 \%$ SDS, $10 \%$ glycerol, and $0,01 \%$ bromophenol blue). The lysates were boiled for $5 \mathrm{~min}$ at $95^{\circ} \mathrm{C}$, centrifuged at $10,000 \times g$ for $5 \mathrm{~min}$ and separated by SDS-PAGE. Proteins were transferred to a PVDF membrane at $120 \mathrm{~V}$ for $80 \mathrm{~min}$ and probed with anti-dBtk antibody (Tsikala et al., 2014) at 1:4000 and anti-syntaxin (Syn) antibody (8C3; Developmental Studies Hybridoma Bank) at 1:3000. Rat and mouse HRP-conjugated antibodies were applied at 1:5000 and proteins were visualized with chemiluminescence (ECL Plus, GE Healthcare). Signals were measured with Bio-Rad Molecular Imager Chemidoc XRS+.

\section{Confocal microscopy}

BtkGal4 flies were crossed to UAS-mCD8-GFP (Lee and Luo, 1999) and progeny were used to examine the expression pattern of $\mathrm{dBtk}$ in the adult brain. Flies were dissected in cold PBS, fixed in $4 \%$ paraformaldehyde for $15 \mathrm{~min}$, and imaged by laser-scanning confocal microscopy (Leica, TCS SP8). Images were captured using a $40 \times / 1.3 \mathrm{NA}$ oil objective after 488 $\mathrm{nm}$ excitation and digital image resolution was set at $1024 \times 1024$. Image stacks were collected at $0.75 \mu \mathrm{m}$ intervals to cover the entire brain. The images were converted to grayscale, inverted in Adobe Photoshop 3, and shown as maximum intensity projections derived from confocal stacks.

\section{Statistical analysis and experimental design}

For all experiments, controls and genetically matched experimental genotypes were tested in the same session in a balanced experimental design. The order of training and testing was randomized. When two genetic controls were used, we required an experimental result to be significantly different from both genetic controls. Untransformed (raw) data were analyzed parametrically with the JMP 7 statistical software package (SAS Institute). If significant, initial ANOVA tests were followed by planned comparisons [least square mean (LSM) contrast analyses] if they indicated significant differences among the genotypes and the level of significance was adjusted for the experiment-wise error rate as suggested by Sokal and Rohlf (1981). 


\section{Results}

dBtk mutants are defective in footshock habituation

In the footshock habituation paradigm (Acevedo et al., 2007), flies avoid the initial 2-8 repetitive stimuli, but their response declines rapidly to an asymptotic baseline as predicted (Rankin et al., 2009), after 10-11 stimuli. This pre-exposuredependent attenuated avoidance after 15 footshocks relative to that of naive animals is quantified as a positive change in the HI of control flies in Figure $1 C$ (open bar). Failure to establish habituation does not attenuate shock avoidance after shock pre-exposure and therefore yields the zero or negative difference from the naive response reported by the HI. Because the HI measures the relative change in avoidance within each genotype, it is not dependent on absolute $45 \mathrm{~V}$ avoidance levels, which can be variable (Table 1).

Viable MiMIC (Venken et al., 2011) insertion mutants $\left(34172-d B t k^{M 1}\right)$ in intron 2 of the $d B t k$ gene (Fig. $1 A$ ), resulting in reduction of both protein isoforms in adult heads (Fig. 1B), did not attenuate shock avoidance following exposure to 15 stimuli, in contrast to controls and heterozygotes, indicating failure to habituate (Fig. $1 C, b t k^{M 1 / M 1}$; ANOVA: $F_{(2,58)}=$ 16.7450, $p<0.0001$; subsequent LSM: $p=1.2 \times 10^{-6}$ vs control). Mutants for a different insertion (34284-btk $k^{M 2}$ ) in intron 4 (Fig. $1 A$ ), which has a milder effect on dBtk levels (Fig. 1B), habituated to the footshocks, albeit not to the same degree as controls (Fig. $1 D, b t k^{M 2 / M 2}$; ANOVA: $F_{(5,68)}=13.3566, p<0.0001$; subsequent LSM: $p=0.0144$ vs control). In agreement with the behavior of $d B t k^{M 1} /+$ (Fig. $1 C, d B t k^{M 1} /+$; ANOVA: $F_{(2,58)}=16.7450$, $p<0.0001$; subsequent LSM: $p=0.4960$ vs control), heterozygotes for a lethal insertion $\left(37042-b t k^{L}\right)$ in intron 4 (Fig. 1A) presented normal habituation (Fig. 1D, $b t k^{L} /+$; ANOVA: $F_{(5,68)}=13.3566, p<$ 0.0001; subsequent LSM: $p=0.4495$ vs control). It should be noted that although the shock AF is intrinsic to calculation of the HI, AFs for all genotypes used herein are collectively presented on Table 1.

To ascertain that the habituation defect is indeed consequent of reduction in dBtk levels and not unrelated mutations on the chromosome, we examined heteroallelic combinations of these independently isolated mutations. Significantly, hetero-allelics of the lethal $b t k^{L}$ with both viable $b t k^{M 1}$ and $b t k^{M 2}$ insertions failed to habituate to 15 footshocks (Fig. 1D; ANOVA: $F_{(5,68)}=13.3566, p<0.0001$; subsequent LSM: $p=1.2 \times 10^{-7} b t k^{M 1 / L}$
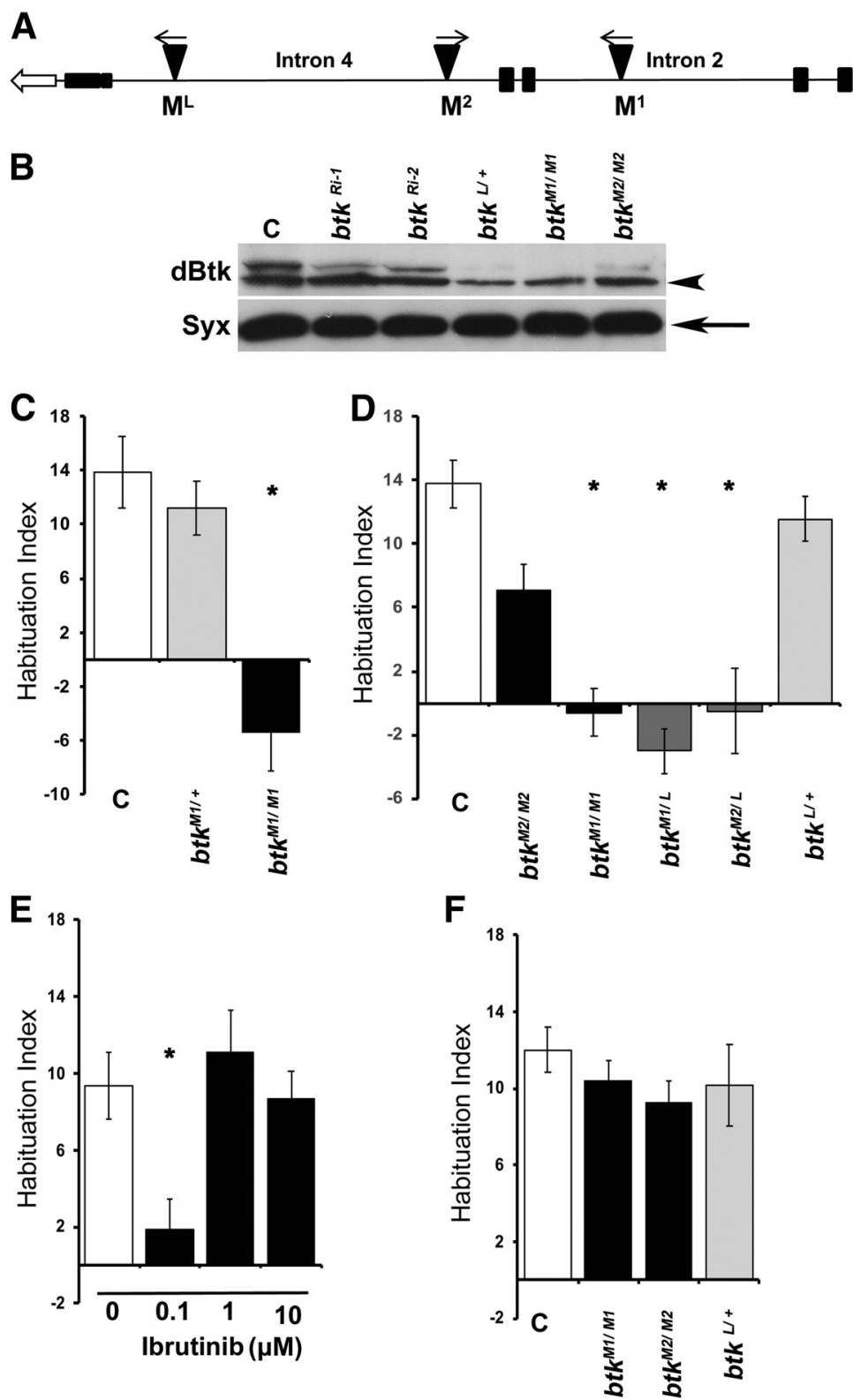

Figure 1. $d B t k$ is essential for footshock habituation. $A, A$ schematic of the $B t k 29 A$ ( $d B t k$ ) genomic area where the transposon insertions used in this study reside. The open arrow demonstrates the direction of transcription. Filled boxes correspond to exons, whereas lines correspond to the indicated introns. The triangles show the MiMIC insertions. $M^{1}$, The homozygous viable MI01270; $M^{2}$, the homozygous viable MI02160; $M^{\mathrm{L}}$, the lethal insertion MI02966. $\boldsymbol{B}$, Western blot analysis of head lysates from five 3- to 5-d-old female btk mutants and pan-neuronally expressing RNAis as indicated. The arrowhead points to a band which migrates as predicted for the long (type 2) dBtk protein, whereas the arrow points to the apparent short (type 1) isoform (Hamada-Kawaguchi et al., 2014). The following strains were used: C, Control ( $\left.y^{1} w^{*}\right) ; b t k^{R i-1}$, pan-neuronal expression under Elav-Gal4 of the BDSC 35159 RNAi-encoding transgene; $b t k^{R i-2}$, pan-neuronal expression under Elav-Gal4 of the BDSC 25791 RNAi-encoding transgene; $b t k^{L /+}$, heterozygotes for the lethal (MI02966) insertion; $b t k^{M 1 / M 1}$, homozygotes for the $M^{1}$ (MI01270) insertion; $b t k^{M 2 / M 2}$, homozygotes for the $M^{2}$ MI02160) insertion. The anti-dBtk antibody (Btk) recognizes two bands, the upper one of which is reduced the most in all mutants and RNAi-expressing animals, with the lower one also reduced, albeit to a lesser degree. Syntaxin 1 (Syx) is used as a loading control. $\boldsymbol{C}-\boldsymbol{F}$, Habituation indices quantify the difference in footshock avoidance following exposure to 15 stimuli from that of same genotype naive flies and are shown as the mean \pm SEM for the indicated number of repetitions $(n)$. Stars indicate significant differences from controls as indicated in the text. $C$, Homozygous bt $k^{M 1 / M 1}$ mutants perform significantly different from mutant heterozygotes and $y^{1} w^{*}$ controls $(\boldsymbol{C}) . n \geq 16$ for all groups. $\boldsymbol{D}$, Complementation of the habituation failure among $d B t k$ insertion mutants. Although the performance of $b t k^{L}$ heterozygotes was not significantly different from that of the $y^{7} w^{*} \operatorname{controls}(\boldsymbol{C})$, heteroallelics of this lethal insertion over both viable M1 and M2 insertions presented significant habituation deficits. $n \geq 9$ for all groups. $\boldsymbol{E}$, The Btk inhibitor Ibrutinib induces habituation deficits in $y^{1} w^{*}$ control flies in a dose-specific manner. 0 represents $y^{1} w^{*}$ animals fed the DMSO vehicle and compared with their performance; $0.1 \mu \mathrm{m}$ Ibrutinib induced a significant deficit, but higher doses did not. $n \geq 9$ for all groups. $\boldsymbol{F}, d B t k$ mutants do not present habituation deficits to 4 min exposure of the aversive odorant OCT. $n \geq 12$ for all groups. 
vs control and $p=1.1 \times 10^{-6} b t k^{M 2 / L}$ vs control), indicating that indeed reduced dBtk in the CNS does not support normal footshock habituation.

Because the human ortholog is implicated in cancers (Tillman et al., 2018) and the kinase domain is highly conserved (Gregory et al., 1987), covalent Btk inhibitors have been developed (Ysebaert and Michallet, 2014) and are available. Hence, dBtk activity was inhibited by feeding the commonly used inhibitor ibrutinib to control flies. Compared with vehicle treated animals, ibrutinib treatment resulted in strong abrogation of footshock habituation at low (Fig. $1 E$; $0.1 \mu \mathrm{M}$, ANOVA: $F_{(3,60)}=5.4217, p=0.0024$; subsequent LSM: $p=0.0012$ vs $0 \mu \mathrm{M})$, but not high concentrations (Fig. $1 E$; ANOVA: $F_{(3,60)}=5.4217, p=0.0024$; subsequent LSM: $p=0.5368,1$ vs $0 \mu \mathrm{M}$, and $p=0.796510$ vs $0 \mu \mathrm{M})$. The reason for this sharp concentration optimum is unclear at the moment, but at higher concentrations it may affect activities of potentially related proteins with opposing effects on habituation to footshock. Nevertheless, the results are consistent with the genetic evidence and strengthen the conclusion that dBtk kinase activity is required for habituation to repeated footshocks. Because, $d B t k$ mutants were found to habituate prematurely in an olfactory jump reflex assay (Asztalos et al., 2007), we tested whether dBtk reduction resulted in deficient olfactory avoidance habituation (Semelidou et al., 2018). However, dBtk abrogation did not affect habituation to aversive odor (OCT) exposure (Semelidou et al., 2018), suggesting that the protein is specifically required for habituation to footshocks (Fig. 1F; ANOVA: $\left.F_{(3,62)}=0.8339, p=0.4806\right)$.

To independently validate these results, we used the TARGET system (McGuire et al., 2003) to abrogate the protein in adult animals by transgene-mediated RNA-interference (RNAi). In fact, adult-specific pan-neuronal (Fig. $2 A$; ANOVA: $F_{(2,44)}=$ $6.1651, p=0.0045$; subsequent LSM: $p=0.0016$ and $p=0.018$ vs controls, respectively), dBtk attenuation recapitulated the footshock habituation failure of the mutants and also did not compromise habituation to the aversive odorant octanol (Fig. 2C; ANOVA: $\left.F_{(2,30)}=1.3588, p=0.2734\right)$. The latter supports the notion that $\mathrm{dBtk}$ functions specifically in footshock habituation. Identical results were obtained with the second RNAi-mediating transgene (Fig. $2 B$; ANOVA: $F_{(2,35)}=11.8202, p<0.0001$; subsequent LSM: $p=0.00068$ and $p=0.00014$ vs controls, respectively) and a different pan-neuronal driver (Gouzi et al., 2011), Ras2Gal4 (Fig. 2D; ANOVA: $F_{(2,27)}=11.2630, p=0.0003$; subsequent LSM: $p=0.0002$ and $p=0.0008$ vs controls, respectively). Therefore, the failed habituation phenotype of $d B t k$ mutants is not developmental in origin, but reflects an acute requirement for dBtk activity to facilitate habituation. Significantly, its abrogation in glia (Fig. 2E; ANOVA: $F_{(2,46)}=0.2643$, $p=0.7690$ ), or constitutively sparing the MBs from dBtk attenuation under MBGal80 (Fig. $2 F$; ANOVA: $F_{(2,47)}=0.0326, p=$ 0.9679; Krashes et al., 2007), did not affect habituation, suggesting that activity of the kinase is required within these neurons. Therefore, the habituation failure is not a consequence merely of the presence of the RNAi encoding transgenes, or drivers, but their induction not in glia, but within MB neurons, as suggested by Figure $2 F$ and reported (Fig. $2 G$ ) expression of dBtk in these neurons.

\section{dBtk functions within $\alpha^{\prime} / \beta^{\prime}$ mushroom body neurons to facilitate footshock habituation}

Neurotransmission from the $\alpha / \beta \mathrm{MB}$ neurons is essential to prevent premature footshock habituation (Acevedo et al., 2007), but apparently not to facilitate its onset. So, we wondered where within the adult CNS is dBtk activity required for facilitation of footshock habituation. Initially, to unequivocally establish that dBtk activity solely within the MBs is essential for facilitation of footshock habituation, we attenuated it therein with the strong pan-MB driver Leo-Gal4 (Messaritou et al., 2009). This abolished footshock habituation (Fig. 3A; ANOVA: $F_{(2,34)}=12.6248, p<$ 0.0001 ; subsequent LSM: $p=0.0001$ and $p=0.0002$ vs controls, respectively) and the result was independently validated with another RNAi transgene (Fig. 3C; ANOVA: $F_{(2,39)}=60,9160, p<$ 0.0001 ; subsequent LSM: $p=1.76 \times 10^{-12}$ and $p=6.55 \times 10^{-10}$ vs controls, respectively), establishing the necessity of $\mathrm{dBtk}$ within the MBs for the process. This conclusion was strengthened by reinstating dBtk specifically within the MBs of adult $b t k^{M 1}$ homozygotes, which fully reversed their deficient habituation (Fig. $3 B$; ANOVA: $F_{(5,66)}=18.3727, p<0.0001$; subsequent LSM: $p=6 \times 10^{-6}$ leoMB-GAL4, btk ${ }^{\mathrm{M} 1} /$ btk $^{\mathrm{Ml}}$; GAL80ts/+ induced vs leoMB-GAL4,btk ${ }^{\mathrm{Ml}} /+$; GAL80ts/ + induced and $p=$

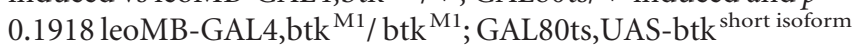
induced vs leoMB-GAL4,btk ${ }^{\mathrm{Ml}} /+$; GAL80ts/+ induced). The transgene encodes the predominately neuronal type 1 short dBtk isoform (Tsikala et al., 2014), containing all conserved domains except the N-terminal PH. However, if the UAS-btk ${ }^{\text {short isoform }}$ was not induced in adult animals the mutant phenotype persisted (Fig. 3B; uninduced LSM: uninduced leoG4,btk $k^{M 1} / b t k^{M 1} ; \mathrm{G}^{\mathrm{ts}} /$ UASbtk $p=0.0367$ vs leoG4,btk ${ }^{M 1} / b t k^{M 1} ; \mathrm{G}^{\mathrm{ts}} /+$ and $p<$ 0.0001 with leoG4,btk $k^{M 1} /+; \mathrm{G} 80^{\mathrm{ts}} /+$ controls, respectively). The acute requirement for $\mathrm{dBtk}$ within adult $\mathrm{MBs}$ for footshock habituation was further supported by attenuating its levels under the pan-MB drivers 247-Gal4 (Fig. 3D; ANOVA: $F_{(2,42)}=$ 11.0752, $p=0.0001$; subsequent LSM: $p=0.0032$ and $p=4 \times$ $10^{-5}$ vs controls, respectively) and dnc-Gal4 (Fig. 3E; ANOVA: $F_{(2,30)}=12.4333, p=0.0001$; subsequent LSM: $p=0.0012$ and $p=0.0001$ vs controls, respectively), both of which precipitated pronounced habituation defects.

Given the role of neurotransmission from $\mathrm{MB} \alpha / \beta$ neurons in preventing premature habituation (Acevedo et al., 2007), we wondered whether dBtk acts within these neurons to inhibit this process and consequently facilitate habituation. Thus, dBtk was attenuated within $\alpha / \beta$ neurons under the $\mathrm{c} 772$ (Fig. 3F; ANOVA: $F_{(2,44)}=0.6731, p=0.5156$ ) and $c 739$ (Fig. $3 G$; ANOVA: $\left.F_{(2,43)}=1.1146, p=0.3378\right)$ drivers in $\alpha \beta$ cortex or core neurons, respectively (Pavlopoulos et al., 2008), but habituation was not altered, eliminating this hypothesis. In addition, dBtk attenuation within $\gamma$ neurons also did not affect footshock habituation (Fig. $3 H$; ANOVA: $F_{(2,47)}=0.0266, p=0.9737$ ). Significantly however, dBtk abrogation within $\alpha^{\prime} / \beta^{\prime}$ MB neurons [Fig. 3I; ANOVA: $F_{(3,44)}=22.5186, p<0.0001$; subsequent LSM: induced (IN) $p<0.0001$ vs both controls] abolished footshock habituation. In contrast, if the RNAi-mediating transgene remained uninduced, habituation was indistinguishable from controls (Fig. 3I; LSM: uninduced (UN) $p=0.0891$ and $p=0.3397$ vs the controls, respectively). Therefore, $\mathrm{dBtk}$ is not developmentally, but rather acutely required within $\alpha^{\prime} / \beta^{\prime} \mathrm{MB}$ neurons to facilitate footshock habituation.

To verify this conclusion independently, we used a "splitGal4" driver expressed specifically in $\alpha^{\prime} / \beta^{\prime} \mathrm{MB}$ neurons and varied the expression of the RNAi-mediating transgene $\left(b t k^{R i-1}\right)$ based on the optimum temperature for Gal4-mediated transcription (McGuire et al., 2004). Raising and maintaining the flies at $18^{\circ} \mathrm{C}$, should not yield appreciable $b t k^{R i-1}$ expression and presented normal habituation within the range of controls (Fig. 1). In contrast, animals raised at $18^{\circ} \mathrm{C}$, but kept at $30^{\circ} \mathrm{C}$ for $48 \mathrm{~h}$ before training to achieve maximal $b t k^{R i-1}$ expression failed to 

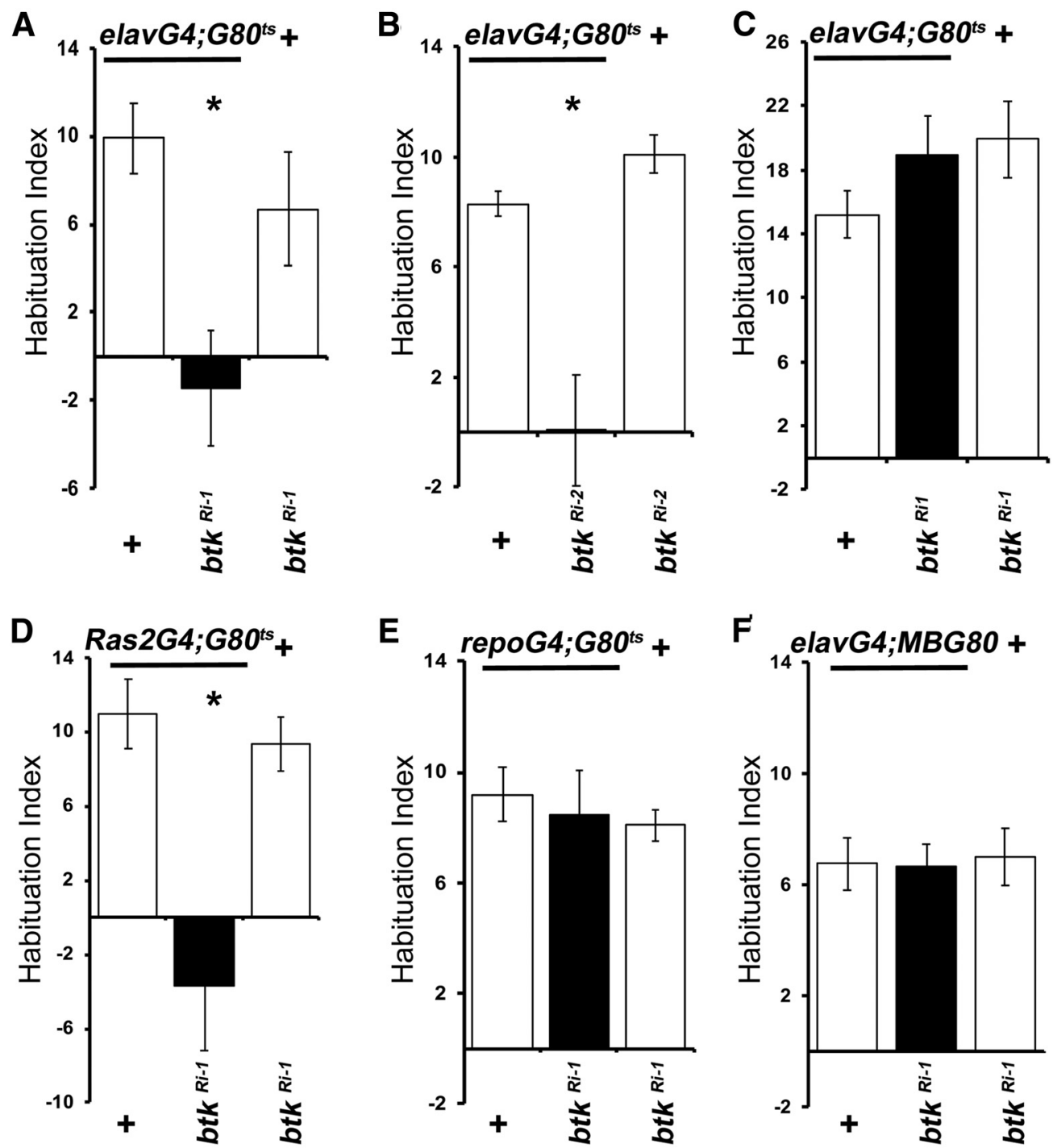

G

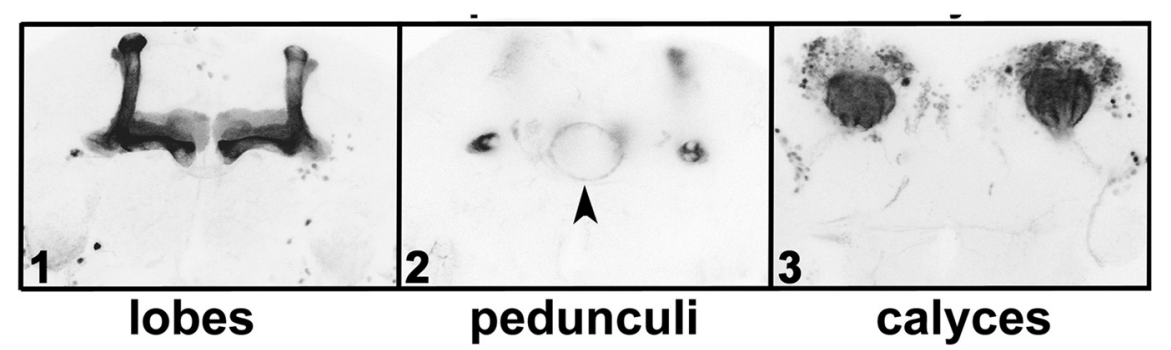

Figure 2. Adult-specific abrogation of dBtk results in deficient footshock habituation. Hls quantifying the difference in footshock avoidance following exposure to 15 stimuli from that of same genotype naive flies are shown as the mean \pm SEM for the indicated number of repetitions $(n)$. All panels show the performance of animals expressing a $d b t k$ RNAi-encoding transgene $\left(b t k^{R i-1} /+\right.$ ) under the indicated driver (black bar), the driver heterozygotes (left open bars), and the RNAi-mediating transgene heterozygotes ( + , right open bars). driver/ + , Progeny from the cross of the $w^{1118}$ background driver with $36303 y^{1} v^{7}$, whereas for the $b t k^{R i-1} /+$, the $y^{1} v^{7}$ background of $b t k^{R i-1}$ was crossed to $w^{1118}$ so that the two controls have equivalent backgrounds as the experimentals. Asterisks indicate significant differences from controls as detailed in the text. $\boldsymbol{A}$, Adult limited pan-neuronal expression of $b t k^{R i-1}$ eliminates habituation to 15 footshocks. $n \geq 11$ for all groups. $\boldsymbol{B}$, Adult-limited pan-neuronal expression of an independent RNAi-mediating transgene ( $\left.b t k^{R i-2}\right)$, also results in habituation defects. $n \geq 9$ for all groups. $\boldsymbol{C}$, Adult-limited pan-neuronal expression of

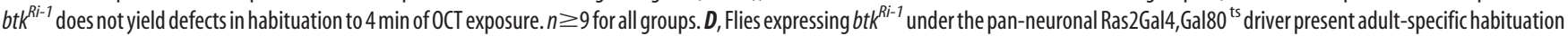
defects compared with controls. $n \geq 9$ for all groups. $\boldsymbol{E}$, Adult limited $b t k^{R i-1}$ expression in glia did not precipitate deficits. $n \geq 14$ for all groups. $\boldsymbol{F}$, Pan-neuronal expression of $b t k^{R i-1}$, but at the exclusion of the MBs did not yield deficits in shock habituation. $n \geq 12$ for all groups. G, dBtk is preferentially distributed within the MBs evidenced by mcD8-GFP expression under the control of the btkG4 driver (BDSC, 49182). The arrowhead indicates faint expression in the ring neurons of the ellipsoid body at the level of the pedunculi.

habituate (Fig. 3J; ANOVA: $F_{(2,55)}=4.8324, p=0.0118$; subsequent LSM: $p=0.0032$ vs $18^{\circ} \mathrm{C}$ ). Footshock habituation in animals raised and kept at $25^{\circ} \mathrm{C}$ was not abolished, but was highly suppressed compared with the performance of animals kept at $18^{\circ} \mathrm{C}$ (Fig. 3J; ANOVA: $F_{(2,55)}=4.8324, p=0.0118$; subsequent LSM: $p=0.0546$ vs $18^{\circ} \mathrm{C}$ ). These results demonstrate that $\mathrm{dBtk}$ function in $\alpha^{\prime} / \beta^{\prime}$ neurons is indeed essential to facilitate footshock habituation. This is not specific to dBtk, as conditionally 

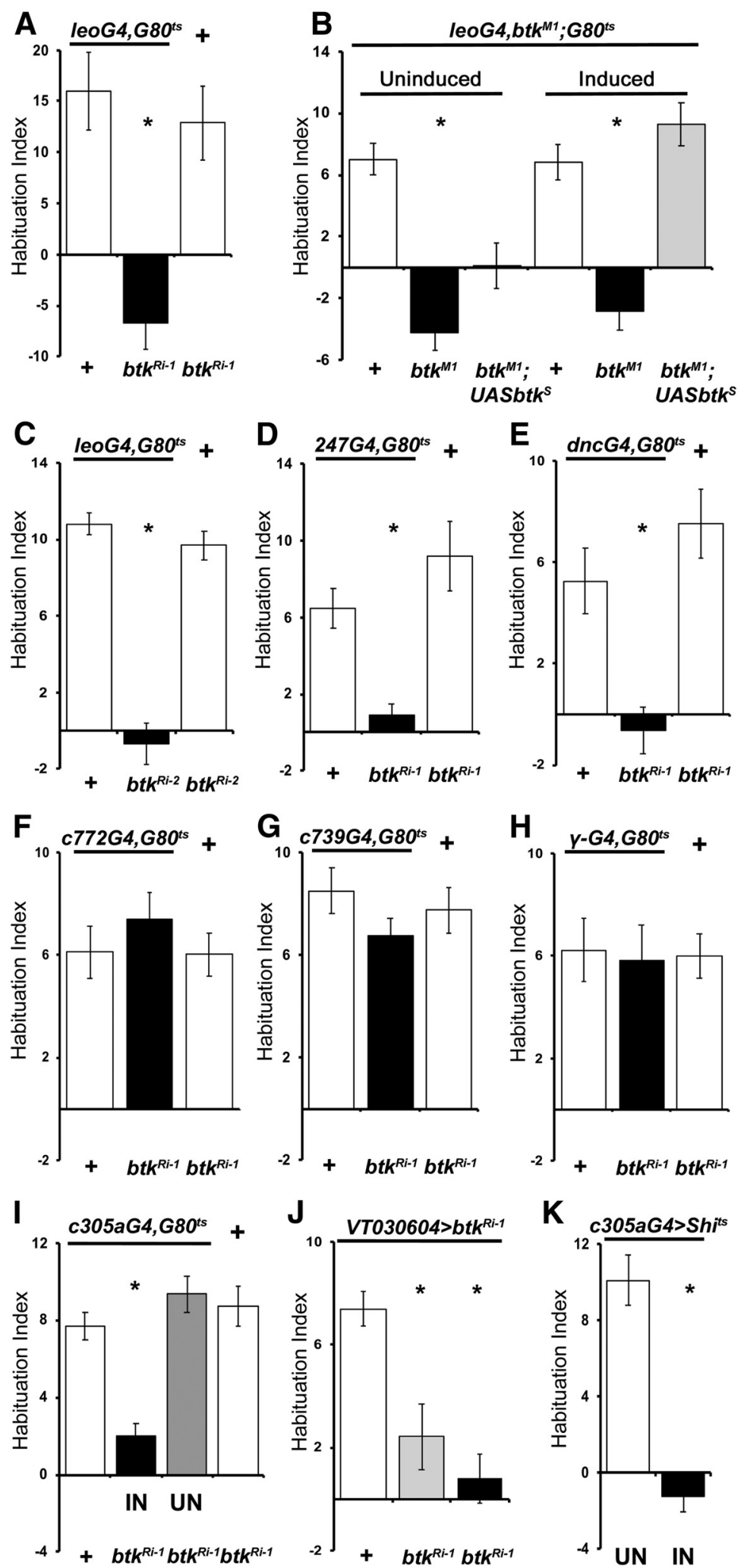

Figure 3. dBtk is acutely required within the $\alpha^{\prime} / \beta^{\prime}$ mushroom body neurons to facilitate habituation to footshock. Habituation indices quantifying the difference in footshock avoidance following exposure to 15 stimuli from that of same genotype naive flies are shown as the mean \pm SEM for the indicated number of repetitions $(n)$. All panels show the performance of animals expressing a dbtk RNAi-encoding transgene $\left(b t k^{R i-1} /+\right)$ under the indicated driver (black bar), the driver heterozygotes (left open bars), and the RNAi-mediating transgene heterozygotes (+, right open bars). driver/ + , Progeny from the cross of the $w^{1118}$ background driver with $36303 y^{7} v^{1}$, whereas for the $b t k^{R i-1} /+$, the $y^{7} v^{7}$ background $b t k^{R i-1}$ was crossed to $w^{1118}$ so that the two controls have equivalent backgrounds as the experimental. Asterisks indicate significant differences from controls as detailed in the text. $A$, Adult limited pan-MB expression of $b t k^{R i-1}$ eliminates habituation to 15 footshocks. $n \geq 11$ for all groups. $\boldsymbol{B}$, Rescue of the habituation deficit of $b t k^{M 1 / M 1}$ by adult-limited expression of a dBtk transgene in the MBs. Uninduced (left side) blocking neurotransmission from $\alpha^{\prime} / \beta^{\prime}$ neurons with the thermosensitive transgenic dynamin Shi ${ }^{\text {ts }}$ (Kitamoto, 2001), under c305a-Gal4 completely blocked habituation (Fig. 3K; ANOVA: $F_{(1,25)}=$ 56.7690, $p<0.0001$; subsequent LSM: $\left.p=9 \times 10^{-8}\right)$. This is despite the small but significant shock avoidance reduction in the "induced" (IN) versus the "uninduced" (UN) state (Table 1), which does not contribute to the phenotype because of the normalization afforded by the calculation of the HI.

The collective results demonstrate two important points. Neurotransmission from $\alpha^{\prime} / \beta^{\prime} \mathrm{MB}$ neurons facilitates footshock habituation, whereas activation of their $\alpha / \beta$ counterparts suppresses it (Acevedo et al., 2007).

dBtk is also required within $\alpha / \beta$ neurons to maintain stimulus responsiveness

Three main reasons prompted us to investigate $\mathrm{dBtk}$ function in $\alpha / \beta$ neurons. The protein appears present in these neurons (Fig. 2G), dbtk mutants habituate prematurely their olfactory jump reflex (Asztalos et al., 2007) and neurotransmission

$\leftarrow$

transgene-bearing flies (gray bar) behave significantly different from controls, much like non-transgene bearing mutants homozygotes $\left(p=0.0014\right.$ and $p=2.2 \times 10^{-6}$, respectively). In contrast, a $2 \mathrm{~d}$ induction of the transgene (gray bar right side) completely reversed the deficient habituation ( $p=$ 0.1918 vs $p=6 \times 10^{-6}$ for mutant homozygotes compare to controls; open bar). $n \geq 8$ for all groups. $C$, Adult limited pan-MB expression of the independent $b t k^{R i-2}$ transgene precipitates defective shock habituation compared with both controls. $n \geq 10$ for all groups. $\boldsymbol{D}$, Adult specific expression of $b t k^{R i-1}$ under the independent pan-MB 247Gal4 driver results in abrogated shock habituation compared with controls. $n \geq$ 14 for all groups. $E$, A third MB-restricted driver (dncGal4) yields adult-specific shock habituation deficits when driving UAS-btk ${ }^{R i-1} . n \geq 9$ for all groups. $\boldsymbol{F}$, Adult-limited $b t k^{R i-1}$ expression in $\alpha / \beta$ MB neurons does not precipitate habituation deficits. $n \geq 11$ for all groups. $G$, Adult-specific $b t k^{R i-1}$ expression in $\alpha / \beta$ MB neurons under the independent $\mathrm{c} 739 \mathrm{Gal} 4$ driver does not compromise shock habituation. $n \geq 13$ for all groups. $\boldsymbol{H}$, Adult-specific $b t k^{R i-1}$ expression in $\gamma \mathrm{MB}$ neurons under VT030604 also does not compromise shock habituation. $n \geq 14$ for all groups. I, Adult-specific IN of $b t k^{R i-1}$ expression in $\alpha^{\prime} / \beta^{\prime}$ MB neurons abrogates shock habituation, whereas the uninduced transgene (UN) does not. $n \geq 9$ for all groups. J, Expression of $b t k^{R i-1}$ driven by the $\alpha^{\prime} / \beta^{\prime}$-specific VT030604 driver present aberrant habituation (black bar) when induced for $2 \mathrm{~d}$ compared to their uninduced siblings. However, when flies were raised at $25^{\circ} \mathrm{C}$ (gray bar), they presented an ameliorated deficit compared with those raised at $18^{\circ} \mathrm{C}$ and uninduced. $n \geq 14$ for all groups. $\boldsymbol{K}$, Expression of shi ${ }^{\text {ts }}$ in $\alpha^{\prime} / \beta^{\prime}$ MB neurons compromises habituation under the restrictive conditions (black bar) compared with animals kept under permissive temperature $\left(p=9 \times 10^{-8}\right)$. $n \geq 11$ for all groups. 
from $\alpha / \beta$ neurons is essential for mediating responsiveness to footshocks and prevent premature habituation (Acevedo et al., 2007). Therefore, we wondered whether dBtk is also involved in maintenance of stimulus responsiveness within $\alpha / \beta$ neurons. Surprisingly, in contrast to controls subjected to the same number of stimuli, $b t k^{M 1}$ mutants habituated prematurely after 6 and 10 footshocks (Fig. 4A; ANOVA: $F_{(5,104)}=34.9318, p<0.0001$; subsequent LSM: 6 shocks $p=4 \times 10^{-10}$, 10 shocks $p=0.0027$ vs controls), but failed to habituate to 15 shocks (LSM: $p=$ $9 \times 10^{-16}$ vs controls). The deficits were phenocopied by acute pan-neuronal dBtk abrogation (Fig. 4B; ANOVA: $F_{(8,156)}=$ 3.8010, $p=0.0004$; subsequent LSM: 6 shocks $p=0.0088$ and $p=0.0193,10$ shocks $p=0.0121$ and $p=0.0259$, 15 shocks $p=0.0057$ and $p=0.0125$ vs controls, respectively) and induced upon inhibition of dBtk activity with Ibrutinib in control flies with a similar dose-response as for failed habituation to 15 stimuli (Fig. 4C; ANOVA: $F_{(2,28)}=10.1984$, $p=0.0005$; subsequent LSM: $p=0.0002$, 0.1 vs $0 \mu \mathrm{M}$ and $p=0.2240,1$ vs $0 \mu \mathrm{M})$. Therefore, as for habituation failure to 15 shocks, the premature avoidance attenuation of the mutants is also not developmental in origin. If the attenuated avoidance is indeed habituation, it should be dishabituated (Rankin et al., 2009). We used an $8 \mathrm{~s}$ puff of $\mathrm{YO}$, which has been shown effective for habituation to aversive odors (Paranjpe et al., 2012; Semelidou et al., 2018). In fact, the premature footshock avoidance attenuation was reversed by post-training exposure to $\mathrm{YO}$ (Fig. 4E; ANOVA: $F_{(4,43)}=45.5267, p<0.0001$; subsequent LSM: 6 shocks $p=1.1 \times 10^{-11}, 10$ shocks $p=1.5 \times 10^{-9}$, AVD after YO $p=1.9 \times 10^{-9}$ ), which also suffices to dishabituate control flies after typical habituation to 15 shock stimuli (Fig. $4 D$; ANOVA: $F_{(2,30)}=84.8182, p<0.0001$; subsequent LSM: YO dishabituation, $p=9.28 \times 10^{-12}$; AVD, $p=6.27 \times$ $\left.10^{-12}\right)$. Because control flies do not habituate to six shocks (Fig. $4 A, B$ ), dishabituation after six shocks was not performed as unnecessary.

Further confirmation that both aberrant habituation phenotypes result from dBtk attenuation and not another mutation on the $b t k^{M 1}$ chromosome was obtained by adult-specific pan-MB abrogation of the protein under Leo-Gal4. This also yielded both premature habituation to 6 and 10 footshocks and failure to habituate to 15 stimuli and significantly both deficits were absent if the attenuating transgene was uninduced (Fig. $5 \mathrm{~A}$; ANOVA: $F_{(5,119)}=8.6993 p<0.0001$; subsequent LSM, 6 shocks, $p=0.0002 ; 10$ shocks, $p=0.0304 ; 15$ shocks, $p=1 \times 10^{-5}$ vs uninduced). Importantly, expression of the dBtk-encoding transgene (UAS- $b t k^{S}$ ) under Leo-Gal4 in $b t k^{M 1}$ homozygotes fully restored both, the ability to prevent premature habituation to 6 and 10 footshocks and to facilitate habituation after 15 stimuli, but not if the transgene remained uninduced (Fig. 5B; and 10 shocks. $n \geq 8$ for all groups.
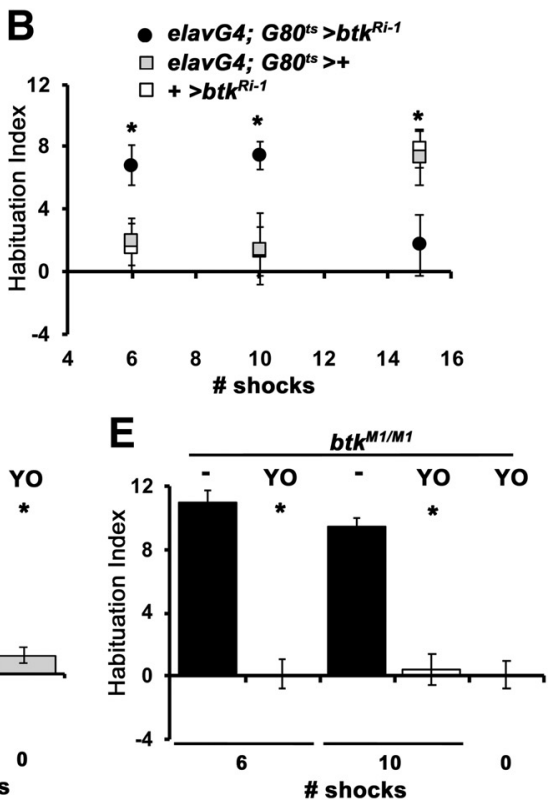

Figure 4. $\mathrm{dBtk}$ is acutely required within $\alpha / \beta \mathrm{MB}$ neurons to inhibit premature habituation to footshock. Habituation indices performance of animals with abrogated dBtk, whole open squares the respective controls. Stars indicate significant differences (10 10 shocks and the habituation failure after 15 stimuli. So that the controls have similar genetic backgrounds, progeny from the cross of the $\mathrm{w}^{1118}$ background ElavGal4;Gal80 ${ }^{\text {ts }}$ with $36303 y^{1} v^{1}$, whereas for the $b t k^{R i-1} /+$, the $y^{1} v^{7}$ background $b t k^{R i-1}$ was crossed to resentation. 0 denotes shock avoidance of naive flies after Y0 exposure. Y0 exposure results in significant dishabituation after 6

ANOVA: $F_{(5,77)}=13.3452 p<0.0001$; subsequent LSM, 6 shocks, $p=0.0036 ; 10$ shocks, $p=0.0161 ; 15$ shocks, $p=6 \times$ $10^{-10}$ vs uninduced). This is consistent with the notion that $\mathrm{dBtk}$ functions to maintain habituation latency within $\alpha / \beta \mathrm{MB}$ neurons and to facilitate footshock habituation within their $\alpha^{\prime} / \beta^{\prime}$ counterparts.

To confirm the independent function of dBtk in both preventing premature habituation in $\alpha / \beta$ and facilitating it in $\alpha^{\prime} / \beta^{\prime}$ neurons, the protein was selectively abrogated therein. Significantly, animals with abrogated $\mathrm{dBtk}$ in adult $\alpha / \beta$ neurons under c739Gal4 habituated prematurely to 6 and 10 stimuli (Fig. $5 C$; ANOVA: $F_{(5,77)}=17.1806, p<0.0001$; subsequent LSM: 6 shocks $p=8.9 \times 10^{-6}, 10$ shocks $p=2.9 \times 10^{-8}, 15$ shocks $p=$ 0.9504 vs control), whereas attenuation in $\alpha^{\prime} / \beta^{\prime}$ neurons under c305aGal4 did not affect habituation latency, but blocked habituation to 15 shocks (Fig. 5D; ANOVA: $F_{(5.82)}=12.2529, p<$ 0.0001 ; subsequent LSM: 6 shocks $p=0.3433,10$ shocks $p=$ $0.777,15$ shocks $p=3.7 \times 10^{-7}$ vs control).

Therefore, dBtk has two functionally distinct and apparently independent neuronal type-specific roles. It engages mechanisms that maintain latency, thus preventing premature habituation within $\alpha / \beta$ neurons and potentially distinct mechanisms that facilitate habituation in their $\alpha^{\prime} / \beta^{\prime}$ counterparts. Alternatively, 

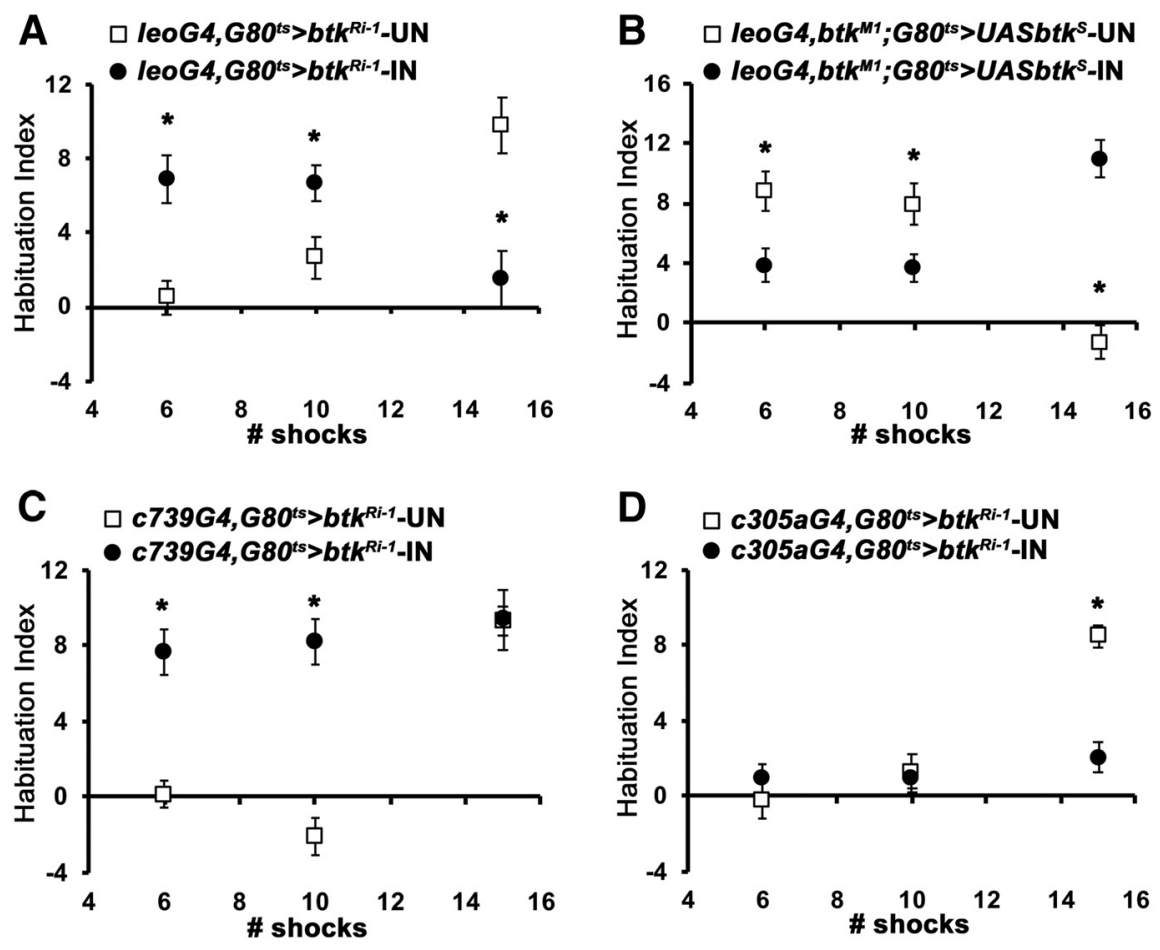

Figure 5. Differential roles for dBtk in latency and habituation in $\alpha / \beta$ and $\alpha^{\prime} / \beta^{\prime} \mathrm{MB}$ neurons. Habituation indices quantifying the difference in footshock avoidance following exposure to the indicated number of stimuli from that of same genotype naive flies are shown as the mean \pm SEM for the indicated number of repetitions $(n)$. Filled circles represent the mean performance of animals with abrogated dBtk; whole open squares, the respective controls. Asterisks indicate significant differences from controls as detailed in the text. $\boldsymbol{A}$, Adult-limited pan-MB expression of $b t k^{R i-1}$ (IN) yields premature habituation after 6 and 10 stimuli and failed habituation after 15 shocks compared to in-genotype controls of siblings with the transgene silent (UN). $n>18$ for all groups. $\boldsymbol{B}$, The complementary experiment of adult-limited pan-MB expression of UAS-btk ${ }^{5}$ rescued (IN) the premature habituation after 6 and 10 stimuli, as well as the habituation deficit after 15 footshocks compared to siblings not expressing the transgene (UN). $n \geq 12$ for all groups. C, Adult-limited expression of $b t k^{R i-1}$ in $\alpha / \beta$ MB neurons (IN) recapitulates the premature habituation after 6 and 10 shocks but not the failed habituation after 15 stimuli, compared to siblings not expressing the transgene (UN). $n \geq$ 10 for all groups. $\boldsymbol{D}$, Adult-limited expression of $b t k^{R i-1}$ in $\alpha^{\prime} / \beta^{\prime}$ MB neurons (IN) recapitulates the failed habituation to 15 footshocks, but not the premature habituation to 6 or 10 stimuli compared to siblings not expressing the transgene (UN). $n \geq 11$ for all groups.

upon activation of these neurons by the footshock stimuli, dBtk may mediate neurotransmission from both types, which in the case of $\alpha / \beta$ neurons is required to maintain the value of the stimulus and prevent premature habituation (Acevedo et al., 2007) and $\alpha^{\prime} / \beta^{\prime}$ neurons to facilitate it (Fig. $3 I, K$ ). Moreover, since $b t k$ mutants present habituation deficits specifically to footshock, but not odor stimuli, it is possible that the kinase is involved in second messenger pathways transducing dopaminergic signals that apparently communicate footshock information to the MBs and result in their activation (Cervantes-Sandoval et al., 2017; Horiuchi, 2019).

\section{Antipsychotics rescue the deficient habituation of dBtk mutants}

We assumed that the inability to habituate upon dBtk attenuation may result from persistent neurotransmission from $\alpha^{\prime} / \beta^{\prime}$. This could be due to excess dopaminergic signaling or inability of the mutants to downregulate the response to dopaminergic input. To differentiate between these possibilities, we initially sought to inhibit dopaminergic inputs pharmacologically. Interestingly, excess dopaminergic signaling has been linked with schizophrenia in humans (Brisch et al., 2014; Kesby et al., 2018), a condition also associated with habituation failures and defects (Gillberg, 2003; Ludewig et al., 2003; Barkus et al., 2014; McDi- armid et al., 2017). In fact, pharmaceuticals used to treat this condition are thought to act mostly as monoamine receptor antagonists including dopaminergic ones (Robinson, 2007; Brunton et al., 2010).

Therefore, to investigate whether monoamine receptors are involved, we sought to antagonize them in the btk mutants, with the typical tricyclic antipsychotic clozapine, thought to primarily address dopamine, but also serotonin receptors and the atypical benzisothiazole risperidone, which is thought to be a more pronounced serotonergic antagonist, but also efficaciously antagonizes additional monoamine receptors (Brunton et al., 2010). Interestingly, administration of clozapine for 16-18 h before habituation training reversed in a dose-dependent manner the inability of $b t k^{M 1}$ homozygotes (Fig. $6 A$; ANOVA: $F_{(2,28)}=11.6054$, $p=0.0002$; subsequent LSM: $5 \mu \mathrm{M} p=$ $0.0041,10 \mu \mathrm{M} p=0.0001$ vs $0 \mu \mathrm{M}$ ) and $b t k^{M 1 / M 2}$ hetero-allelics (Fig. 6B; ANOVA: $F_{(2,30)}=4.7128, p=0.0172$; subsequent LSM: $5 \mu \mathrm{M} p=0.0648,10 \mu \mathrm{M} p=0.0048$ vs $0 \mu \mathrm{M}$ ) to habituate to 15 footshocks. A similar treatment with risperidone also reversed the defective habituation of $b t k^{M 1}$ homozygotes, although at the high concentration of $10 \mu \mathrm{M}$, the effect of the drug appeared reduced (Fig. 6C; ANOVA: $F_{(3,44)}=9.0647, p=0.0001$; subsequent LSM: $0.1 \mu \mathrm{M} p=0.0005,1 \mu \mathrm{M} p=1.2 \times$ $10^{-5}, 10 \mu \mathrm{M} p=0.0066$ vs $\left.0 \mu \mathrm{M}\right)$. Assuming that clozapine antagonizes dopamine receptors, dBtk loss appears to affect their levels or activity. Since phenotypic reversal was elicited by risperidone, at least at the lower concentrations and serotonergic neurotransmission has not been reported to mediate footshock information to the MBs, it is likely that this drug also addresses dopaminergic receptors.

Importantly, clozapine treatment also reversed the defective footshock habituation of animals with pan-MB RNAi-mediated dBtk abrogation (Fig. 6D; ANOVA: $F_{(2,26)}=5.2147, p=0.0132$; subsequent LSM: $5 \mu \mathrm{M} p=0.0531,10 \mu \mathrm{M} p=0.0038$ vs $0 \mu \mathrm{M}$ ) suggesting that the drug reaches these neurons and mediates the observed phenotypic reversal upon acute dBtk attenuation therein. Significantly, both pharmaceuticals reversed the habituation defect to 15 shocks in animals with dBtk abrogation specifically in $\alpha^{\prime} / \beta^{\prime}$ MB neurons (Fig. 6E; ANOVA: $F_{(2,27)}=19.1763$, $p<0.0001$; subsequent LSM: $10 \mu \mathrm{M}$ clozapine $p<0.0001,10 \mu \mathrm{M}$ risperidone $p<0.0001$ vs untreated-0). As expected, dBtk attenuation in $\alpha / \beta$ neurons did not yield deficient habituation, and the drugs did not affect normal habituation to 15 footshocks (Fig. 6F; ANOVA: $\left.F_{(2,26)}=1.5268, p=0.2376\right)$. These results confirm that the habituation failure is specifically driven by $\mathrm{dBtk}$ attenuation within the $\alpha^{\prime} / \beta^{\prime}$ neurons and likely results from consequent excess or elevated signaling from one or more monoamine receptors within these neurons. In fact, Dop1R2 (Crittenden et al., 1998) and 5HT1B have been reported to be expressed in these neurons (Ries et al., 2017). Moreover, dBtk abrogation 

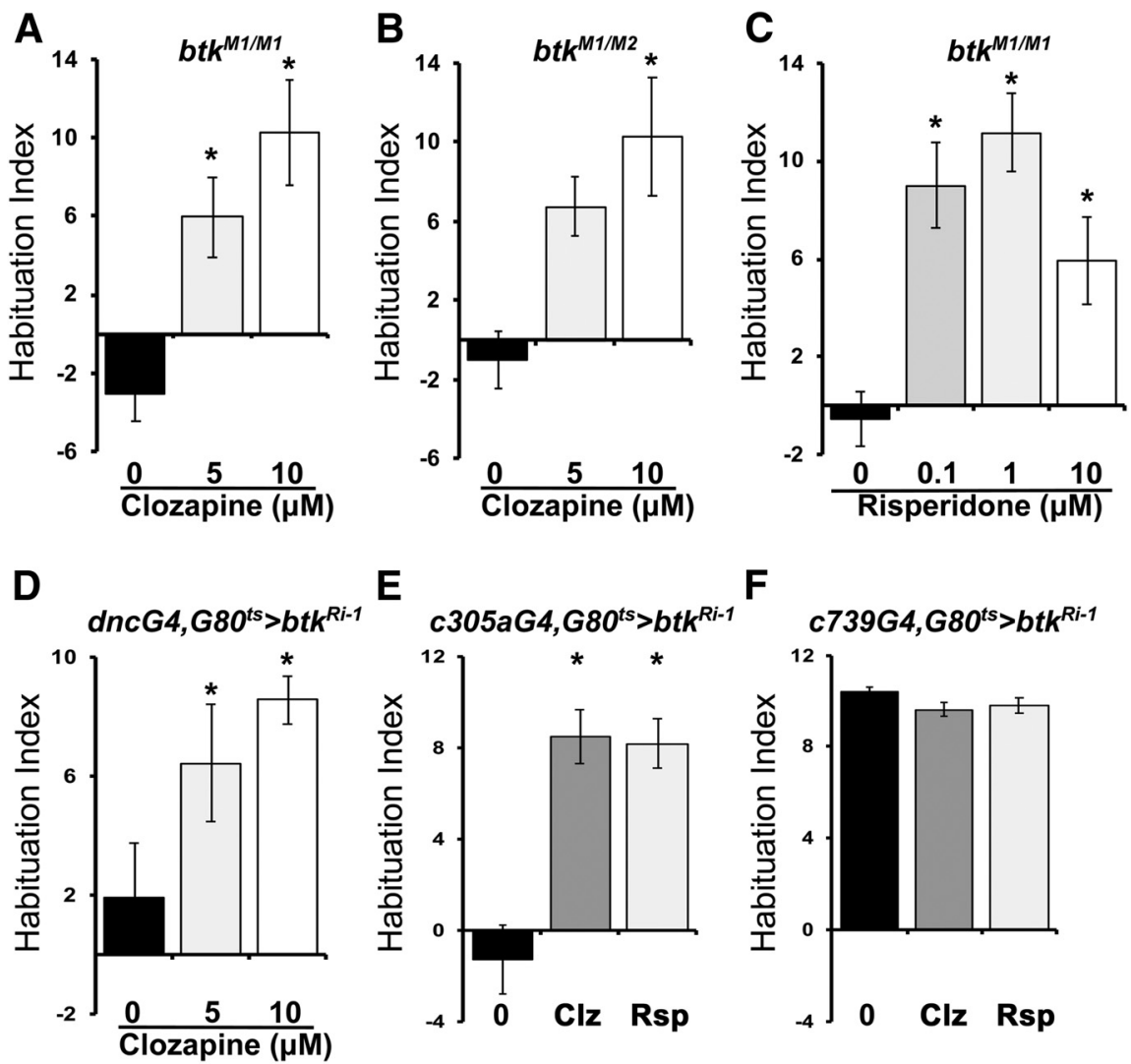

E

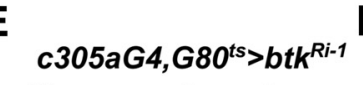

\section{$\mathbf{F}$}
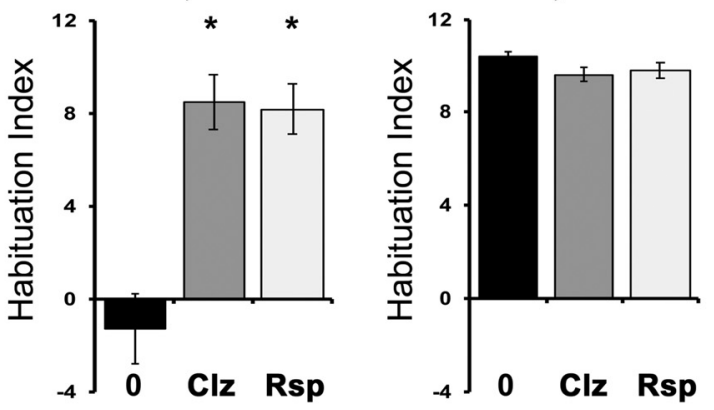

G

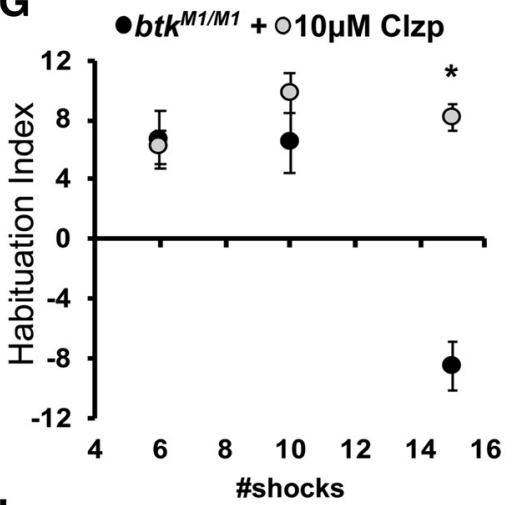

I

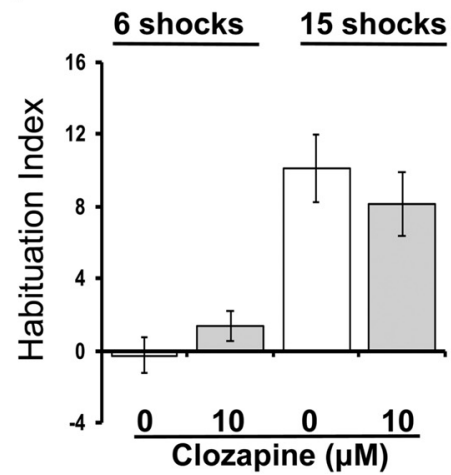

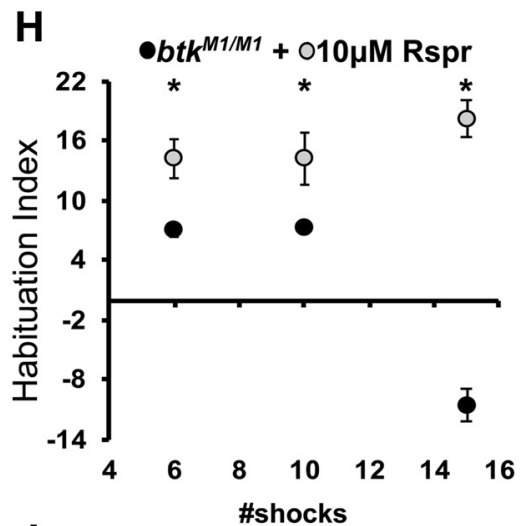

J

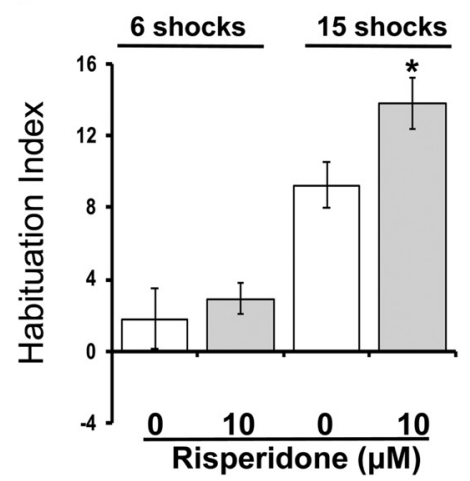

Figure 6. Antipsychotics selectively rescue the defective habituation of btk mutants. Habituation indices quantifying the difference in footshock avoidance following exposure to the indicated number of stimuli from that of same genotype naive flies are shown as the mean \pm SEM for the indicated number of repetitions $(n)$. Asterisks indicate significant differences from controls as detailed in the text. $\boldsymbol{A}$, Clozapine restores the defective habituation of $b t k^{M 1}$ homozygotes. Compared with vehicle-treated mutants that do not habituate to 15 footshocks (0), habituation was significantly improved after treatment with 5 and $10 \mu \mathrm{M}$ clozapine. $n \geq 9$. $\boldsymbol{B}$, Clozapine restores the defective habituation of $b t k^{M 1 / M 2}$ hetero-allelics, which present a strong habituation defect if treated only with vehicle (0). Clozapine at 5 and $10 \mu \mathrm{m}$ restored habituation to 15 footshocks. $n \geq 8$ for all groups

in $\alpha / \beta$ neurons did not affect habituation to 15 shocks, further suggesting that the drugs specifically address consequences of dBtk abrogation within their $\alpha^{\prime} / \beta^{\prime}$ counterparts.

As $b t k$ mutants present both impaired latency and failed habituation, we wondered whether the antipsychotics can reverse both phenotypes. Interestingly, 10 $\mu \mathrm{M}$ clozapine did not alter the premature habituation of $b t k^{M 1}$ homozygotes, but potently reversed their failure to habituate (Fig. $6 G$; ANOVA: $F_{(5,95)}=7.8245, p<$ 0.0001 ; subsequent LSM: 6 shocks $p=$ $0.8085,10$ shocks $p=0.1382,15$ shocks $p=7.3 \times 10^{-7}$ vs control). Risperidone also potently suppressed the habituation failure in the mutants (Fig. $6 \mathrm{H}$; ANOVA: $F_{(5,73)}=39.2195, p<0.0001$; subsequent LSM: 6 shocks $p=0.00014,10$ shocks $p=$ $0.0017,15$ shocks $p=1 \times 10^{-19}$ vs control), but interestingly it increased significantly the shock avoidance of the mutants (Table 1), suggesting that it may actually induce hyperactivity, increased locomotion or enhance the aversion of the $45 \mathrm{~V}$ shock. In agreement, whereas $10 \mu \mathrm{M}$ clozapine did not affect avoidance (Table 1), or habituation to 6 or 15 stimuli in control flies (Fig. 6I; ANOVA: $F_{(3,52)}=$ 14.7186, $p<0.0001$; subsequent LSM: 6 shocks $p=0.3096,15$ shocks $p=0.3398$ vs control), risperidone $(10 \mu \mathrm{M})$ consistently elevated $45 \mathrm{~V}$ avoidance in control

C, Risperidone restores the defective habituation of $b t k^{M 1}$ homozygotes. The strong habituation defect to 15 shocks of vehicle-treated mutant homozygotes was reversed after treatment with $0.1,1$, and $10 \mu \mathrm{m}$ risperidone. $n \geq 9$ for all groups. $\boldsymbol{D}$, The habituation defect precipitated by adultlimited pan-MB abrogation of $\mathrm{dBtk}$ is barely reversible by 5 $\mu \mathrm{M}$, but restored with $10 \mu \mathrm{M}$ clozapine compared with vehicle-treated animals $(0) . n \geq 8$ for all groups. $\boldsymbol{E}$, Clozapine $(10 \mu \mathrm{M})$ and risperidone $(10 \mu \mathrm{m})$ restore the defective habituation phenotype precipitated by adult-limited abrogation of $\mathrm{dBtk}$ in $\alpha^{\prime} / \beta^{\prime}$ neurons. $n \geq 9$ for all groups. $\boldsymbol{F}$, Clozapine (10 $\mu \mathrm{m})$ and risperidone $(10 \mu \mathrm{m})$ do not affect the performance of flies where adult-limited abrogation of $\mathrm{dBtk}$ in $\alpha / \beta$ neurons. $n \geq 9$ for all groups. I, Clozapine $(10 \mu \mathrm{m})$ in control flies does not induce premature habituation after 6 shocks, or a change in habituation after 15 such stimuli relative to vehicle (0)treated controls. $n \geq 9$ for all groups.J, Risperidone $(10 \mu \mathrm{M})$ in control flies does not induce premature habituation after 6 shocks, but yields elevated habituation after 15 such stimuli relative to vehicle (0)-treated controls. $n \geq 9$ for all groups. $\mathbf{G}$, Clozapine selectively restores the defective, but not the premature habituation of $b t k^{\mathrm{M} 1}$ homozygotes. Clozapine did not change the premature habituation to 6 or 10 stimuli, but reversed the habituation failure after 15 footshocks. $n \geq 10$ for all groups. $\boldsymbol{H}$, Risperidone restores the defective habituation, but also enhances the premature habituation of $b t k^{\mathrm{M} 1}$ homozygotes. Risperidone rescued the habituation defect after 15 stimuli, but also resulted in significantly higher habituation scores after 6 and 10 footshocks. $n \geq 10$ for all groups. 
flies (Table 1). However, this did not affect habituation to 6 shocks in control animals, but appeared to mildly enhance habituation after 15 stimuli (Fig. 6F; ANOVA: $F_{(3,53)}=16.9637, p<$ 0.0001; subsequent LSM: 6 shocks $p=0.5796,15$ shocks $p=$ 0.0205 vs control). Collectively these results suggest distinct mechanisms of action for the two antipsychotics in Drosophila, in agreement with their proposed activities in vertebrates. In fact, risperidone is a potent serotonin and dopamine antagonist, but unlike clozapine presents measurable activities against adrenergic and histamine receptors (PDSD Ki database; Roth et al., 2000), which may underlie the increase in avoidance or locomotion upon its administration.

\section{Discussion}

The response dynamics to repeated footshocks define two phases. Avoidance is initially maintained for $8-10$ stimuli, followed by its rapid attenuation to an apparently asymptotic habituated response by the $15^{\text {th }}$ stimulus (Acevedo et al., 2007; Figs. $4 A, B$, $5 A, B$, controls). Neurotransmission from $\alpha / \beta \mathrm{MB}$ neurons is required to maintain stimulus responsiveness in the initial latency phase (Acevedo et al., 2007) and significantly we now demonstrate that synaptic activity from their $\alpha^{\prime} / \beta^{\prime}$ counterparts is essential to facilitate the subsequent habituation phase (Fig. $2 K$ ). Therefore, the two habituation phases are mediated by distinct MB neurons.

Because neurotransmission from different $\mathrm{MB}$ neurons is required both to prevent and to induce the habituated response, it is likely that it engages distinct $\alpha / \beta$ and $\alpha^{\prime} / \beta^{\prime}$ MB output neurons (MBONs; Takemura et al., 2017). We hypothesize that these potentially antagonistic signals are relayed to neurons driving the choice to avoid or ignore the footshocks in a manner akin to neurons toggling attractive and aversive odor responses in Drosophila (Yamazaki et al., 2018). It is unclear at the moment how the activities of the two types of $\mathrm{MB}$ neurons are coordinated upon repeated stimulation as indicated by the response dynamics (Acevedo et al., 2007; Fig. 3A, $C-F$ ). However, the current data predict that synaptic transmission from $\alpha / \beta$ neurons requisite for maintenance of habituation latency precedes $\alpha^{\prime} / \beta^{\prime}$ activation, which facilitates habituation onset. Nevertheless, how neurotransmission from $\alpha / \beta$ neurons is attenuated after a relatively set number of stimuli and why it precedes activation of $\alpha^{\prime} / \beta^{\prime}$ neurons is unclear at the moment. Significantly, abrogation of $\mathrm{dBtk}$ in adult $\alpha / \beta$ neurons yielded strong latency attenuation (Fig. $3 E$ ), whereas loss from $\alpha^{\prime} / \beta^{\prime}$ neurons did not affect latency, but eliminated habituation (Fig. $3 F$ ). Therefore, although $\alpha / \beta$ and $\alpha^{\prime} / \beta^{\prime}$ activities may be coordinated, they appear independent.

Importantly, we describe the first mutant with specific defects in footshock habituation and demonstrate that dBtk activity is acutely required in adult $\mathrm{MBs}$ for both phases (Figs. $1 B, C, 3 A$ ), in accord with its apparent expression within $\alpha / \beta$ and $\alpha^{\prime} / \beta^{\prime}$ neurons. The premature habituation upon $\mathrm{dBtk}$ abrogation suggests reduced or dysregulated neurotransmission from $\alpha / \beta$ neurons (Acevedo et al., 2007; Fig. $2 \mathrm{~K}$ ), whereas the habituation defect is consistent with attenuated neurotransmission from their $\alpha^{\prime} / \beta^{\prime}$ counterparts. As Btk is involved in regulation of actin cytoskeleton dynamics (Corneth et al., 2016) and given the involvement of cortical actin in neurotransmitter release (Rust and Maritzen, 2015), the kinase may modulate directly or indirectly the responsiveness to afferent signaling and subsequent neurotransmission to $\mathrm{MBONs}$ from both types of $\mathrm{MB}$ neurons. In agreement with this notion, $\mathrm{dBtk}$ loss specifically from $\alpha / \beta$ and $\alpha^{\prime} / \beta^{\prime}$ neurons phenotypically mimics Shibire ${ }^{\text {ts }}$-dependent silencing of their synaptic output (Figs. 3I-K, 5C,D).

Alternatively, dBtk may be involved in footshock signal reception at least by the $\alpha^{\prime} / \beta^{\prime}$ neurons, which specifically respond to the dopamine and serotonin receptor antagonists clozapine and risperidone upon attenuation of the kinase. Given that footshock signals are relayed to the MBs by dopamine (Cognigni et al., 2018) and these neurons contain at least one dopamine (Crittenden et al., 1998) and serotonin (Ries et al., 2017) receptors, dBtk loss may alter the number or activity of these receptors within these neurons. Interestingly, mammalian Btk is implicated in regulation of G-protein-coupled receptor (GPCR) signaling (Corneth et al., 2016) and importantly, clozapine and risperidone address and antagonize primarily the typical serotonin and dopamine GPCRs (Naheed and Green, 2001; Brunton et al., 2010). This is in accord with the notion that in $\alpha^{\prime} / \beta^{\prime}$ neurons $\mathrm{dBtk}$ negatively regulates dopamine and/or serotonin receptor signaling or levels. It follows that dBtk loss would increase the levels or activity of one or more of these receptors, altering $\mathrm{MB}$ activation threshold and reducing synaptic transmission to efferents mediating the habituated response. Clozapine and risperidone-mediated antagonism of this putative $\alpha^{\prime} / \beta^{\prime}$ overactivation upon repetitive footshock may restore regulated neurotransmission mediating normal habituation. In contrast, in $\alpha / \beta$ neurons, which do not appear to respond to dopamine or serotonin receptor antagonism, dBtk may regulate neurotransmitter release via its modulation of actin dynamics (Corneth et al., 2016). Impaired neurotransmission from $\alpha / \beta$ neurons may also underlie the reduced latency to suppress the olfactory jump response of $b t k$ mutants (Asztalos et al., 2007).

Therefore, we propose that dBtk may differentially regulate neuronal activities in $\alpha / \beta$ and $\alpha^{\prime} / \beta^{\prime}$ neurons. In $\alpha / \beta$ by positively regulating neurotransmitter release, which is impaired upon dBtk loss, leading to shortened latency and premature habituation. In contrast, in $\alpha^{\prime} / \beta^{\prime}$ neurons dBtk could have primarily a postsynaptic role, by negatively regulating the number or downstream signaling of dopamine and/or serotonin receptors. Elevated intra- $\alpha^{\prime} / \beta^{\prime}$ monoamine receptor signaling upon dBtk loss may lead to dysregulation of downstream synaptic activity and functional silencing of neurotransmission required to facilitate footshock habituation onset. Ongoing experiments aim to test these hypotheses.

It is intriguing that schizophrenia patients also present habituation defects manifested as failures in prepulse inhibition (Braff et al., 2001; Akdag et al., 2003; Ludewig et al., 2003; Meincke et al., 2004; van Os and Kapur, 2009), where a weak prestimulus inhibits the reaction to a following strong startling stimulus. These defects are thought to reflect inability to devalue inconsequential stimuli (Braff et al., 2001; Swerdlow et al., 2008) and can be reversed with antipsychotics including clozapine and risperidone implicating excessive or dysregulated dopaminergic and/or serotonergic signaling similar to $b t k$ mutants. Recent genome-wide association studies (GWASs) suggested linkage of polymorphisms in a number of genes to schizophrenia and other neuropsychiatric disorders (Schizophrenia Working Group of the Psychiatric Genomics Consortium, 2014; Fromer et al., 2016; Roussos et al., 2016), but human Btk was not among them. Nevertheless, given that habituation deficits in flies and humans are reversible with antipsychotics, it is possible that mutations in Drosophila orthologs of genes linked to schizophrenia by GWAS may also present defective footshock habituation and provide expedient experimental validation of their effects on signaling within and between CNS neurons. 


\section{References}

Acevedo SF, Froudarakis EI, Kanellopoulos A, Skoulakis EM (2007) Protection from premature habituation requires functional mushroom bodies in Drosophila. Learn Mem 14:376-384.

Akdag SJ, Nestor PG, O’Donnell BF, Niznikiewicz MA, Shenton ME, McCarley RW (2003) The startle reflex in schizophrenia: habituation and personality correlates. Schizophr Res 64:165-173.

Aso Y, Grübel K, Busch S, Friedrich AB, Siwanowicz I, Tanimoto H (2009) The mushroom body of adult Drosophila characterized by GAL4 drivers. J Neurogenet 23:156-172.

Asztalos Z, Baba K, Yamamoto D, Tully T (2007) The fickle mutation of a cytoplasmic tyrosine kinase effects sensitization but not dishabituation in Drosophila melanogaster. J Neurogenet 21:59-71.

Baba K, Takeshita A, Majima K, Ueda R, Kondo S, Juni N, Yamamoto D (1999) The Drosophila Bruton's tyrosine kinase (Btk) homolog is required for adult survival and male genital formation. Mol Cell Biol 19:4405-4413.

Barkus C, Sanderson DJ, Rawlins JN, Walton ME, Harrison PJ, Bannerman DM (2014) What causes aberrant salience in schizophrenia? A role for impaired short-term habituation and the GRIA1 (GluA1) AMPA receptor subunit. Mol Psychiatry 19:1060-1070.

Braff DL, Geyer MA, Swerdlow NR (2001) Human studies of prepulse inhibition of startle: normal subjects, patient groups, and pharmacological studies. Psychopharmacology 156:234-258.

Brisch R, Saniotis A, Wolf R, Bielau H, Bernstein HG, Steiner J, Bogerts B, Braun AK, Jankowski Z, Kumaritlake J, Henneberg M, Gos T (2014) The role of dopamine in schizophrenia from a neurobiological and evolutionary perspective: old fashioned, but still in vogue. Front Psychiatry 5:47.

Brunton L, Chabner B, Knollman B (2010) Goodman and Gilman's the pharmacological basis of therapeutics, Ed 12. New York: McGraw Hill.

Cervantes-Sandoval I, Phan A, Chakraborty M, Davis RL (2017) Reciprocal synapses between mushroom body and dopamine neurons form a positive feedback loop required for learning. eLife 6:e23789.

Cognigni P, Felsenberg J, Waddell S (2018) Do the right thing: neural network mechanisms of memory formation, expression and update in Drosophila. Curr Opin Neurobiol 49:51-58.

Schizophrenia Working Group of the Psychiatric Genomics Consortium (2014) Biological insights from 108 schizophrenia-associated genetic loci. Nature 511:421-427.

Corneth OB, Klein Wolterink RG, Hendriks RW (2016) BTK signaling in B cell differentiation and autoimmunity. Curr Top Microbiol Immunol 393:67-105.

Crittenden JR, Skoulakis EM, Han KA, Kalderon D, Davis RL (1998) Tripartite mushroom body architecture revealed by antigenic markers. Learn Mem 5:38-51.

D'Cruz AM, Ragozzino ME, Mosconi MW, Shrestha S, Cook EH, Sweeney JA (2013) Reduced behavioral flexibility in autism spectrum disorders. Neuropsychology 27:152-160.

Fromer M, Roussos P, Sieberts SK, Johnson JS, Kavanagh DH, Perumal TM, Ruderfer DM, Oh EC, Topol A, Shah HR, Klei LL, Kramer R, Pinto D, Gümüş ZH, Cicek AE, Dang KK, Browne A, Lu C, Xie L, Readhead B, et al. (2016) Gene expression elucidates functional impact of polygenic risk for schizophrenia. Nat Neurosci 19:1442-1453.

Gai Y, Liu Z, Cervantes-Sandoval I, Davis RL (2016) Drosophila SLC22A transporter is a memory suppressor gene that influences cholinergic neurotransmission to the mushroom bodies. Neuron 90:581-595.

Gillberg C (2003) Deficits in attention, motor control and perception: a brief review. Arch Dis Child 88:904-910.

Gouzi JY, Moressis A, Walker JA, Apostolopoulou AA, Palmer RH, Bernards A, Skoulakis EM (2011) The receptor tyrosine kinase Alk controls neurofibromin functions in Drosophila growth and learning. PLoS Genet 7:e1002281.

Gouzi JY, Bouraimi M, Roussou IG, Moressis A, Skoulakis EMC (2018) The Drosophila Receptor Tyrosine Kinase Alk Constrains Long-Term Memory Formation. J Neurosci. 38:7701-7712.

Gregory RJ, Kammermeyer KL, Vincent WS 3rd, Wadsworth SG (1987) Primary sequence and developmental expression of a novel Drosophila melanogaster src gene. Mol Cell Biol 7:2119-2127.

Guven-Ozkan T, Davis RL (2014) Functional neuroanatomy of Drosophila olfactory memory formation. Learn Mem 21:519-526.

Hamada-Kawaguchi N, Yamamoto D (2017) Ovarian polarity and cell shape determination by Btk29A in Drosophila. Genesis 55:e23042.
Hamada-Kawaguchi N, Nore BF, Kuwada Y, Smith CI, Yamamoto D (2014) Btk29A promotes Wnt4 signaling in the niche to terminate germ cell proliferation in Drosophila. Science 343:294-297.

Horiuchi J (2019) Recurrent loops: incorporating prediction error and semantic/episodic theories into Drosophila associative memory models. Genes Brain Behav 2019:e12567.

Jansiewicz EM, Newschaffer CJ, Denckla MB, Mostofsky SH (2004) Impaired habituation in children with attention deficit hyperactivity disorder. Cogn Behav Neurol 17:1-8.

Kalita J, Bhoi SK, Misra UK (2014) Is lack of habituation of evoked potential a biological marker of migraine? Clin J Pain 30:724-729.

Kesby J, Eyles D, McGrath J, Scott J (2018) Dopamine, psychosis and schizophrenia: the widening gap between basic and clinical neuroscience. Transl Psychiatry 8:30.

Kitamoto T (2001) Conditional modification of behavior in Drosophila by targeted expression of a temperature-sensitive shibire allele in defined neurons. J Neurobiol 47:81-92.

Krashes MJ, Keene AC, Leung B, Armstrong JD, Waddell S (2007) Sequential use of mushroom body neuron subsets during Drosophila odor memory processing. Neuron 53:103-115.

Lee T, Luo L (1999) Mosaic analysis with a repressible cell marker for studies of gene function in neuronal morphogenesis. Neuron 22:451-461.

Ludewig K, Geyer MA, Vollenweider FX (2003) Deficits in prepulse inhibition and habituation in never-medicated, first-episode schizophrenia. Biol Psychiatry 54:121-128.

Massa J, O'Desky IH (2012) Impaired visual habituation in adults with ADHD. J Atten Disord 16:553-561.

Mattsson P, Vihinen M, Smith C (1996) ) X-linked agammaglobulinemia (XLA): a genetic tyrosine kinase (Btk) disease. Bioessays 18:825-834.

McDiarmid TA, Bernardos AC, Rankin CH (2017) Habituation is altered in neuropsychiatric disorders: a comprehensive review with recommendations for experimental design and analysis. Neurosci Biobehav Rev 80: $286-305$.

McGuire SE, Le PT, Osborn AJ, Matsumoto K, Davis RL (2003) Spatiotemporal rescue of memory dysfunction in Drosophila. Science 302:17651768 .

McGuire S, Roman G, Davis R (2004) Gene expression systems in Drosophila: a synthesis of time and space. Trends Genet 20:384-391.

Meincke U, Light GA, Geyer MA, Braff DL, Gouzoulis-Mayfrank E (2004) Sensitization and habituation of the acoustic startle reflex in patients with schizophrenia. Psychiatry Res 126:51-61.

Messaritou G, Leptourgidou F, Franco M, Skoulakis EM (2009) A third functional isoform enriched in mushroom body neurons is encoded by the Drosophila 14-3-3zeta gene. FEBS Lett 583:2934-2938.

Naheed M, Green B (2001) Focus on clozapine. Curr Med Res Opin 17:223229.

Paranjpe P, Rodrigues V, VijayRaghavan K, Ramaswami M (2012) Gustatory habituation in Drosophila relies on rutabaga (adenylate cyclase)dependent plasticity of GABAergic inhibitory neurons. Learn Mem 19: 627-635.

Pavlopoulos E, Anezaki M, Skoulakis EM (2008) Neuralized is expressed in the $\alpha / \beta$ lobes of adult Drosophila mushroom bodies and facilitates olfactory long-term memory formation. Proc Natl Acad Sci U S A 105:1467414679.

Ramaswami M (2014) Network plasticity in adaptive filtering and behavioral habituation. Neuron 82:1216-1229.

Rankin C, Abrams T, Barry R, Bhatnagar S, Clayton D, Colombo J, Coppola G, Geyer M, Glanzman D, Marsland S, McSweeney F, Wilson D, Wu CF, Thompson R (2009) Habituation revisited: an updated and revised description of the behavioral characteristics of habituation. Neurobiol Learn Mem 92:135-138.

Ries AS, Hermanns T, Poeck B, Strauss R (2017) Serotonin modulates a depression-like state in Drosophila responsive to lithium treatment. Nat Commun 8:15738.

Robinson D (2007) CNS receptor partial agonists: a new approach to drug discovery. Primary Psychiatry 14:22-24.

Roth BL, WK Kroeze WK, S Patel S, Lopez E (2000) The multiplicity of serotonin receptors: uselessly diverse molecules or an embarrasment of riches? Neuroscientist 6:252-262.

Roulier E, Panzer S, Beckendorf SK (1998) The Tec29 tyrosine kinase is required during Drosophila embryogenesis and interacts with Src64 in ring canal development. Mol Cell 1:819-829. 
Roussos PG, Zouraraki C, Fullard JF, Karagiorga VE, Tsapakis EM, Petraki Z, Siever LJ, Lencz T, Malhotra A, Spanaki C, Bitsios P (2016) The relationship of common risk variants and polygenic risk for schizophrenia to sensorimotor gating. Biol Psychiatry 79:988-996.

Rust MB, Maritzen T (2015) Relevance of presynaptic actin dynamics for synapse function and mouse behavior. Exp Cell Res 335:165-171.

Semelidou O, Acevedo S, Skoulakis EM (2018) Temporally specific engagement of distinct neuronal circuits regulating olfactory habituation in Drosophila. eLife 7:e39569.

Shyu WH, Chiu TH, Chiang MH, Cheng YC, Tsai YL, Fu TF, Wu T, Wu CL (2017) Neural circuits for long-term water-reward memory processing in thirsty Drosophila. Nat Commun 8:15230.

Siniatchkin M, Kropp P, Gerber WD (2003) What kind of habituation is impaired in migraine patients? Cephalalgia 23:511-518.

Sokal RR, Rohlf FJ (1981) Biometry the principles and practice of statistics in biological research, Ed 2. New York: WH Freeman.

Sunouchi K, Koganezawa M, Yamamoto D (2016) Requirement of the Tec family tyrosine kinase Btk29a for courtship memory in Drosophila males. Arch Insect Biochem Physiol 91:165-174.

Swerdlow NR, Weber M, Qu Y, Light GA, Braff DL (2008) Realistic expectations of prepulse inhibition in translational models for schizophrenia research. Psychopharmacology 199:331-388.

Takemura SY, Aso Y, Hige T, Wong A, Lu Z, Xu CS, Rivlin PK, Hess H, Zhao T, Parag T, Berg S, Huang G, Katz W, Olbris DJ, Plaza S, Umayam L, Aniceto R, Chang LA, Lauchie S, Ogundeyi O, et al. (2017) A connectome of a learning and memory center in the adult Drosophila brain. eLife 6:e26975.
Tei S, Fujino J, Hashimoto R, Itahashi T, Ohta H, Kanai C, Kubota M, Nakamura M, Kato N, Takahashi H (2018) Inflexible daily behaviour is associated with the ability to control an automatic reaction in autism spectrum disorder. Sci Rep 8:8082.

Thompson R, Spencer W (1966) Habituation: a model phenomenon for the study of neuronal substrates of behavior. Psychol Rev 73:16-43.

Tillman B, Pauff J, Satyanarayana G, Talbott M, Warner J (2018) Systematic review of infectious events with the Bruton tyrosine kinase inhibitor ibrutinib in the treatment of hematologic malignancies. Eur J Haematol 100:325-334.

Tsikala G, Karagogeos D, Strigini M (2014) Btk-dependent epithelial cell rearrangements contribute to the invagination of nearby tubular structures in the posterior spiracles of Drosophila. Dev Biol 396:42-56.

van Os J, Kapur S (2009) Schizophrenia. Lancet 374:635-645.

Venken KJ, Schulze KL, Haelterman NA, Pan H, He Y, Evans-Holm M, Carlson JW, Levis RW, Spradling AC, Hoskins RA, Bellen HJ (2011) MiMIC: a highly versatile transposon insertion resource for engineering Drosophila melanogaster genes. Nat Methods 8:737-743.

Yamazaki D, Hiroi M, Abe T, Shimizu K, Minami-Ohtsubo M, Maeyama Y, Horiuchi J, Tabata T (2018) Two parallel pathways assign opposing odor valences during Drosophila memory formation. Cell Rep 22:23462358.

Ysebaert L, Michallet AS (2014) Bruton's tyrosine kinase inhibitors: lessons learned from bench-to-bedside (first) studies. Curr Opin Oncol 26:463468. 